\title{
Disconnection of Intracortical Synaptic Linkages Disrupts Synchronization of a Slow Oscillation
}

\author{
F. Amzica and M. Steriade \\ Laboratoire de Neurophysiologie, Faculté de Médecine, Université Laval, Quebec, Canada G1K 7P4
}

The intracortical synaptic linkages underlying the synchronization of a recently described slow $(<1 \mathrm{~Hz})$ oscillation (Steriade et al., 1993b,c) were investigated in anesthetized cats by means of multisite extra- and intracellular recordings, including dual impalements, from rostral and caudal sites in the association cortical suprasylvian and marginal gyri, before and after reversible lidocaine inactivation or transections in the middle suprasylvian gyrus. Stimulusevoked responses revealed that the rostral and caudal suprasylvian foci are reciprocally connected, with a preference for posterior-to-anterior responses. Lidocaine infusion between the stimulating and recording sites disrupted the intracortical synaptic linkage, while leaving unaffected the responses at the sites close to the stimulating electrodes. The high coherence between slowly oscillating field potentials and intracellular activities recorded from anterior and posterior suprasylvian foci was lost after reversible inactivation or transections in the middle suprasylvian gyrus, whereas the synchrony between adjacent foci within the anterior or posterior areas was preserved. Two to four hours after inactivation or transection the synchrony between all channels was totally or partially recovered. We introduced the synchrony coefficient (SyCo) and calculated the SyCo for closely located and distant sites. Lidocaine infusion or transection did not affect the SyCo between leads placed on the same site, but significantly $(60 \%)$ decreased the SyCo between channels separated by the functionally inactivated or transected sector.

Our results demonstrate that pathways within or beneath the suprasylvian gyrus sustain the synchronization of the slow oscillation between cortical sites. As the loss of longrange coherence was not permanent, intergyral paths and/ or corticothalamocortical loops may exert compensatory functions after the disconnection of intrasuprasylvian synaptic linkages.

[Key words: association cortex, intracortical synaptic linkages, slow oscillation, synchronization, intracellular recordings, field potentials, cross-correlations, EEG]

A slow oscillation, with sequences consisting of depolarizinghyperpolarizing components and recurring periodically at a frequency of $0.1-1 \mathrm{~Hz}$, was described in intracellular recordings

\footnotetext{
Received Nov. 28, 1994; revised Jan. 19, 1995; accepted Jan. 20, 1995.

This work was supported by a grant from the Medical Research Council of Canada (MT-3689). F.A. is a doctoral student partially supported by a fellow ship from the Fonds de la Recherche en Santé du Québec. We thank G. Oakson, P. Giguère, and D. Drolet for technical assistance.

Correspondence should be addressed to Professor Dr. M. Steriade at the above address.

Copyright $\bigcirc 1995$ Society for Neuroscience $0270-6474 / 95 / 154658-20 \$ 05.00 / 0$
}

from neocortical areas in anesthetized or undrugged brainstemtransected cats and, at the EEG level, in naturally sleeping cats and humans (Steriade et al., 1993b,c). The two other EEG rhythms during the state of resting sleep are spindles $(7-14 \mathrm{~Hz})$, occurring in early sleep stages, and delta waves $(1-4 \mathrm{~Hz})$ that progressively replace spindles with the deepening of sleep (Steriade, 1993). The newly discovered slow cortical oscillation at $0.1-1 \mathrm{~Hz}$ is reflected in thalamic reticular and thalamocortical neurons and has the virtue of grouping within periodically recurring wave sequences the thalamic-generated spindles and clock-like delta oscillation as well as the cortical-generated delta waves (Steriade et al., 1993c,d).

The neuronal synchronization underlying the slow oscillation was investigated by means of multisite, extra- and intracellular recordings from visual, association, and motor cortical areas of anesthetized cats (Amzica and Steriade, 1995). The results, based on analyses of membrane potential fluctuations, unit discharges, and field potentials, demonstrated coherent activities with mean time lags of about $10 \mathrm{msec}$ between closely located (1-2 Inm) neurons within the same area, 20-40 nnsec between adjacent areas, but as long as $120 \mathrm{msec}$ in distant recordings from motor and visual cortices. While relatively short time lags may be ascribed to direct or oligosynaptic connections between cortical areas, the long time lags probably implicate inhibitionrebound sequences within the cortex or corticothalamocortical loops. Indeed, dual intracellular recordings from cortical and related thalamocortical neurons showed that the low-threshold spike-bursts of thalamic cells, which follow the corticothalamic volley and are projected back to cortex, may be delayed by 100 msec or more (Contreras and Steriade, 1995).

The above set of results raised the question whether or not intracortical pathways are necessary and sufficient for the synchronization of the slow oscillation. Although previous experiments demonstrated the presence of singly oscillating neurons after thalamectomy (Steriade et al., 1993c), the intracortical synchronization processes remained to be studied by attempting to disconnect various cortical foci, to investigate the slow rhythm after the disruption of synaptic linkages between various cortical sites, and to determine the time course of the effects. The present experiments used multisite recordings including dual impalements of distantly located cortical neurons, reversible and irreversible procedures to inactivate intracortical pathways, and demonstrate that the intracortical disconnection is followed by a long-lasting, but not permanent, loss of the long-range synchronization of the slow oscillation.

\section{Materials and Methods}

The experiments were performed on adult cats anesthetized with ketamine and xylazine (10-15 mg/kg and 2-3 mg/kg, i.m.). The tissues to be 
incised and pressure points were infiltrated with lidocaine. The animals were paralyzed with gallamine triethiodide and artificially ventilated. The end-tidal $\mathrm{CO}_{2}$ concentration (3.5-3.8\%), rectal temperature (37$38^{\circ} \mathrm{C}$ ), and heartbeat were continuously monitored. The EEG was continuously monitored throughout the experiments in order to maintain a constant sleep-like state and additional doses of anesthetics were administered at the earliest tendency toward an activated pattern. The stability of the recordings was improved by bilateral pneumothorax, cisternal drainage, hip suspension, and by filling the hole made in the calvarium with $4 \%$ agar dissolved in saline.

At the end of the experiments, the animals were deeply anesthetized (sodium pentobarbital, $50 \mathrm{mg} / \mathrm{kg}$ ) and perfused transcardially with physiological saline followed by $10 \%$ paraformaldehyde. The brain was removed and stored in formalin with $30 \%$ sucrose, sectioned at $80 \mu \mathrm{m}$, and stained with thionine for histological control of stimulating and recording electrodes as well as of the extension of intracortical transections.

Recordings and stimulation. The recording setup consisted of two groups of electrodes placed in the anterior and posterior parts of the suprasylvian and/or marginal gyrus. Each group contained microelectrodes and several (two to eight) EEG electrodes. Intracellular recordings were obtained by means of glass micropipettes filled with a $3 \mathrm{M}$ solution of $\mathrm{K}$ acetate (impedance, 25-35 M 2 ). A high-impedance dual anplifier (for double intracellular recordings) with active bridge circuitry was used to record and inject current into neurons. EEG activity was recorded bipolarly with coaxial electrodes. The ring was placed at the cortical surface and the tip at a depth of $0.6-0.8 \mathrm{~mm}$.

Stimulation consisted of short $(0.1-0.3 \mathrm{msec})$ pulses $(0.05-0.8 \mathrm{~mA})$ delivered through coaxial electrodes. All signals were digitally recorded on tape (bandpass, DC to $9 \mathrm{kHz}$ ) and fed into a computer for off-line analysis.

The disconnection between the two recording foci was obtained either through reversible inactivation or through transection. The functional inactivation was achieved with microinjections of lidocaine (10-20\%, $1-10 \mu \mathrm{l})$ through a Iamilton syringe inserted into the middle suprasylvian gyrus, between the rostral and caudal recording sites, at a depth of $1 \mathrm{~mm}$. The liquid was injected at a rate of $4 \mu \mathrm{l} / \mathrm{min}$. The inactivation and the extent of its spread was assessed by recording the EEG with an array of two to eight coaxial electrodes $(1 \mathrm{~mm}$ interelectrode distance), placed close to the cannula. In other experiments, thin cortical transections were performed with an ophthalmologic blade after burning the pial surface with silver nitrate.

Data analysis. The fluctuations in the membrane potential of intracellularly recorded neurons and local field potentials (FPs) are regarded as time series. FPs were derived from either extracellular recordings (low-filtered between D.C. and $100 \mathrm{~Hz}$, to avoid spikes) or from EEG recordings (same bandpass). Synchronization was measured by means of correlation techniques. As defined by Bendat and Piersol (1980), a cross-correlation function between two waves may contain a central peak whose amplitude, between -1 and 1 , provides information about the degree of resemblance and the phase sign of the two waves, while the abscissa of the peak yields to the time lag separating the two waves. The cross-correlation function also contains information about the common spectral features of the underlying waves.

Our correlation analyses also highlighted the dynamic evolution of the synchronization between multisite recordings. Figure 1 explains the protocol we developed for a sequential correlation analysis. Consider the dynamic synchronization between waves $X$ and $Y$. These activities may derive from intracellularly recorded postsynaptic potentials (PSPs), FPs (as in Fig. 1), or any ohler tine series. As shown elsewhere (Steriade et al., 1993b; Amzica and Steriade, 1995), the cortical activity during sleep or anesthesia is dominated by a slow oscillation $(<1 \mathrm{~Hz})$. This event is retlected in FPs by a wave complex centered on a sharp deflection which is negative in the cortical depth, preceded by a positive dome, and followed by a rising slope indented by spindles $(7-14 \mathrm{~Hz})$ or faster oscillations (Contreras and Steriade, 1995). For this reason we decided to divide long blocks of data (minimum $2 \mathrm{~min}$, containing at least 60 cycles of the slow oscillation) into sequential windows $\left(\sigma_{1}, \sigma_{2}\right.$, etc.) of $2 \mathrm{sec}$ each. The procedure consisted in the automatic detection of the depth-negative peaks in one channel and the extraction of waves of $2 \mathrm{sec}$ length ( $\pm 1 \mathrm{sec}$ around the peak time) from all simultaneously recorded channels.

Each couple of waves in each window yields to a cross-correlation function (middle row in Fig. 1: $\rho_{\mathrm{X} 1 Y 1}, \rho_{\mathrm{X} 2 \mathrm{Y} 2}$, etc.). Further, the set of cross-correlations generates a three-dimensional surface (3-D). The ab- scissa corresponds to the time lags, the ordinate indicates the ordinals of cross-correlations and is therefore an equivalent of time, and the $\mathrm{z}$-axis displays the correlation coefficients. The gray scaling, in this and all similar figures of this article, assigns white to peaks close to 1 and darkens the shades as values approach -1 (see also scaling bar in Fig. 1). A continuously synchronized couple of waves will produce a scenery with well aligned, high (white) peaks.

A top view of the 3-D sequence of correlations produces the contour map. It is generated by uniting with a line all points with the same height and by shading correspondingly (same calibration bar as for 3-D) the inner part of the closed curve. Contour maps show mainly the alignment of peaks (or valleys) and their latencies. The evolution of maxima from each cross-correlation may also be displayed as a sequence of dots relative to a zero-time lag ( $\tau$ in Fig. 1).

In order to provide a more quantitative measure for the degree of synchrony between two sites we introduced the measure of the Synchrony Coefficient (SyCo). It is derived from a sequence of cross-correlations by calculating the average time lag $\left(\tau_{\text {avg }}\right)$ of the sequence of cross-correlations and the height of each cross-correlation at $\tau_{\text {avg. }}$. SyCo is the average of the previously calculated heights. It is known that the amplitude of a cross-correlation ranges between -1 and 1 . Therefore, SyCo will also range between -1 and 1 . A SyCo of 1 means a perfect synchrony, with all peaks of maximal amplitude and strictly aligned, a SyCo of -1 stands for a perfect synchrony in phase opposition, while a SyCo equal to zero denotes a lack of dynamic synchrony. In our experiments, the SyCo was useful to outline the loss of synchrony between two cortical sites after their functional disconnection.

The rhythmicity and the spectral content of the recordings were obtained by applying fast Fourier transforms (FFT) to the auto and/or cross-correlation functions. It is known (Wiener-Kinchin theorem) that the spectral density functions derived from rough data are identical to the corresponding functions defined in terms of Fourier transforms of correlation functions (Bendat and Piersol, 1980). In order to differentiate between the frequencies that are common for two channels and those expressed independently, we calculated the difference between the cross-spectrum (FFT of the cross-correlation) and the autospectrum (FFT of the autocorrelation) of one channel. The negative components (see dotted parts, marked by arrow, in Fig. 9) indicate frequencies that are specific to the respective channel and are not expressed in the companion channel.

\section{Results}

\section{Data base}

Multisite field activity was recorded in 34 sessions. Additionally, in some experiments we made double intracellular impalements (17 couples) or recorded the intracellular activity of one neuron simultaneously with one or several extracellular unit discharges (10 couples). The membrane potential of somatically impaled cells was more negative than $-60 \mathrm{mV}$ (without current) and the amplitude of their action potentials was higher than $60 \mathrm{mV}$ (but see Fig. 3B). Lidocaine infusions were performed in nine animals. In four cats we made coronal cuts in the middle of the suprasylvian gyrus or performed larger intracortical transections extending from the marginal to the ectosylvian gyri. Each recording session underwent a complete analysis in order to disclose the synaptic linkages and the pattern of synchrony between each lead.

\section{Electrophysiological identification of intracortical suprasylvian pathways}

The existence of functional pathways that would be able to synchronize pools of neurons in the suprasylvian gyrus was investigated by using the experimental design shown in the scheme of Figure 2. The rostral part of the cat's suprasylvian gyrus (I) comprises areas 5a and 5b (Hassler and Muhs-Clement, 1964). The area 7 lies in the middle part of the gyrus (2). The posterior part (3) includes the caudal sector of area 7 as well as visual areas 21 and 19 (Sherk, 1986). For further details on the complex organization of the posterolateral suprasylvian region, also 
Figure 1. Algorithm of analysis for sequential field correlation. Two channels of data, $X$ and $Y$, are sliced in successive windows $\sigma_{1}, \sigma_{2}, \ldots, \sigma_{10}$. Each window extracts $2 \mathrm{sec}$ of each wave and is centered on a negative peak detected in the reference channel ( $Y$ in this case). The resulting couples from each window yield to the cross-correlation functions $\rho_{\mathrm{X} 1 Y 1}, \rho_{\mathrm{X} 2 Y_{2}}, \ldots, \rho_{\mathrm{X} 10 Y 10}$. Unfolding the cross-correlations provides a three-dimensional (3-D) surface. The abscissa represents the time lags between waves $X$ and $Y$, the ordinate is the equivalent of time (one point corresponding to a window), and the $\mathrm{z}$-axis provides the strength of the correlation. The calibration convention (bar depicted at right) attributes white to high, in phase correlation; black to high, but opposite phase correlation; and intermediate gray scales for values between -1 and 1 . Zero values are for lack of correlation. The 3-D surface may be presented as a contour map as well. This is a projection of the $3-D$ surface on the abscissa-ordinate plane in which points of the same height are united with lines and the space enclosed in this contour is shadowed with the corresponding gray scale. Roughly, a contour map is a top view of the $3-D$ surface. The right bottom panel $\tau$ displays the sequence of time lags between the two waves in a window. Each point represents the abscissa of the highest peak of the respective cross-correlation. This graph summarizes the dispersion of the correlation peaks around zero time lag (dotted line).

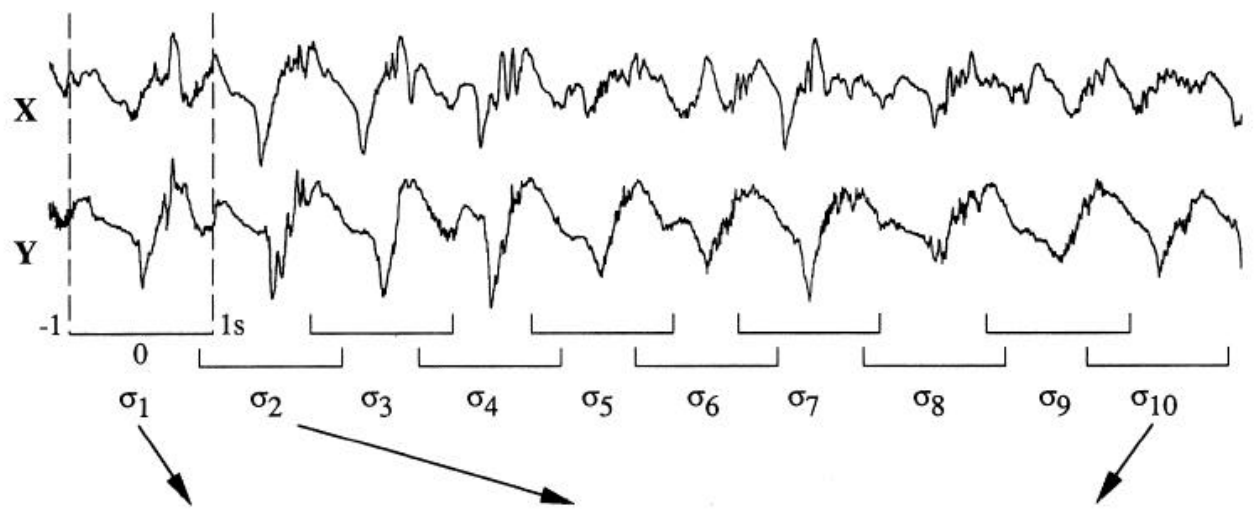

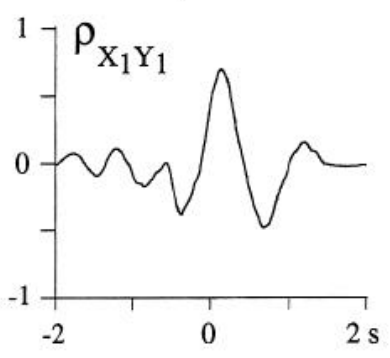
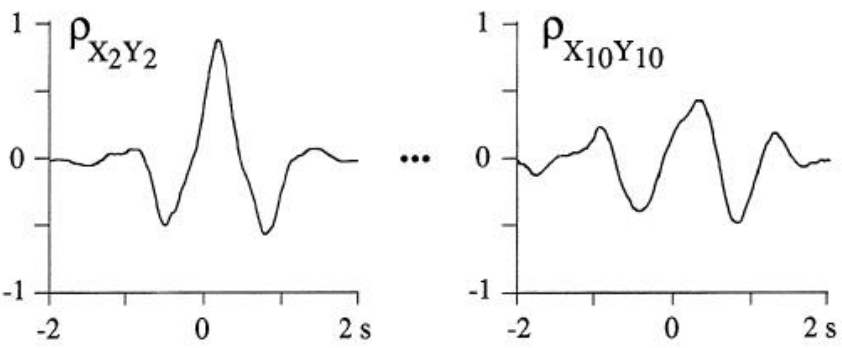

Contour map

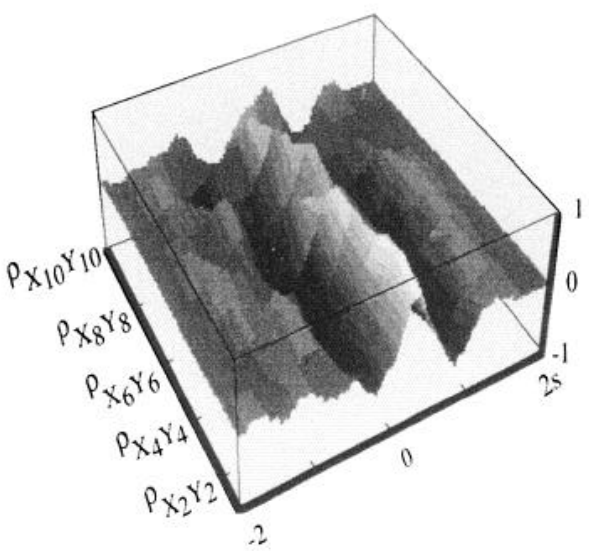

$\tau$

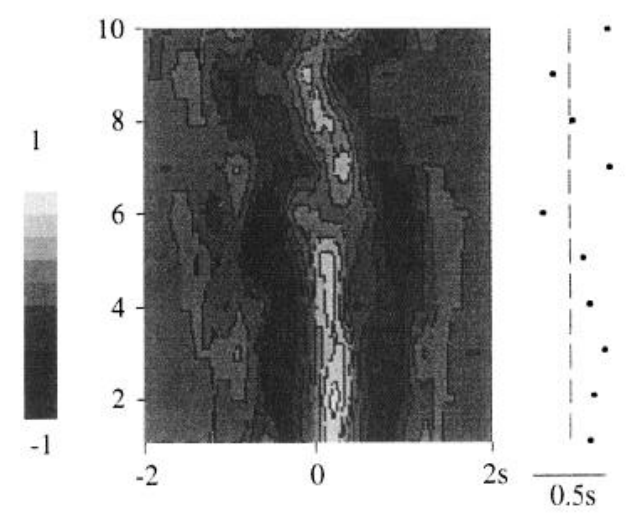

termed the Clare-Bishop (1954) area, see Heath and Jones (1971) and Montero (1981). Two coaxial electrodes (one for stimulation and one for FP recording) and a glass pipette for intracellular recording were inserted in the rostral and the caudal suprasylvian gyrus. The distance between neighboring electrodes in each of these areas was about $1 \mathrm{~mm}$. The distance between the rostral and caudal sites is about $15 \mathrm{~mm}$.

Stimulus-evoked responses show that the two (rostral and caudal) suprasylvian sites are reciprocally connected. (1) Stimulation through the anterior coaxial electrode (Fig. $2 A$ ) elicited an antidromic spike in the closely located cell (A1). An EPSP was also revealed by hyperpolarizing the membrane at $-70 \mathrm{mV}$. The cell excitation was reflected as a biphasic depth negativity in the FP, with a similar time course. Generally, the anterior stimulation was less effective in evoking responses in the middle and caudal recording sites (compared to posterior-to-anterior responses), as shown by the progressively reduced amplitude of FPs and barely visible intracellular response ( $A 2$ and $A 3$ ). (2) Stimuli through the posterior electrode (Fig. $2 B$ ) induced EPSPs in both caudal and rostral cells. The posterior one responded at a shorter latency ( $3 \mathrm{msec}, \mathrm{B3})$ than the anterior one $(7 \mathrm{msec}, B 1)$. The FP re- cordings reflected the same increase in latency with the distance, while the amplitude was comparable. The mean latencies $\pm \mathrm{SE}$ of synaptic responses derived from our intracellular recordings in 17 couples are $3.4 \pm 0.75 \mathrm{msec}$ for local stimulation, and $10.2 \pm 3.45 \mathrm{msec}$ for distant stimulation.

Repetitive cortical stimulation (three to five volleys at $10 \mathrm{~Hz}$ ) elicited incremental responses in distantly located foci. These were characterized by an increase in the amplitude and duration of late EPSPs (Fig. 3Al). In the average of 15 individual responses (Fig. 3A2), the decaying slope of the response induced by the first stimulus of the pulse-train (open arrow) was superimposed on the augmenting response to the second and third stimuli (solid arrow). Thus, the surface in black represents the contribution of the augmenting response. The incremental responses may arise from corticothalamic interactions, but they can also be generated intracortically (see Discussion).

The incrementing response depicted in Figure $3 B$ belongs to a presumed intradendritic recording because (1) the membrane potential, without current, was $-78 \mathrm{mV}$, as measured relative to the potential recorded when leaving the cell, while the heights of action potentials were relatively low $(25-30 \mathrm{mV})$, and three 


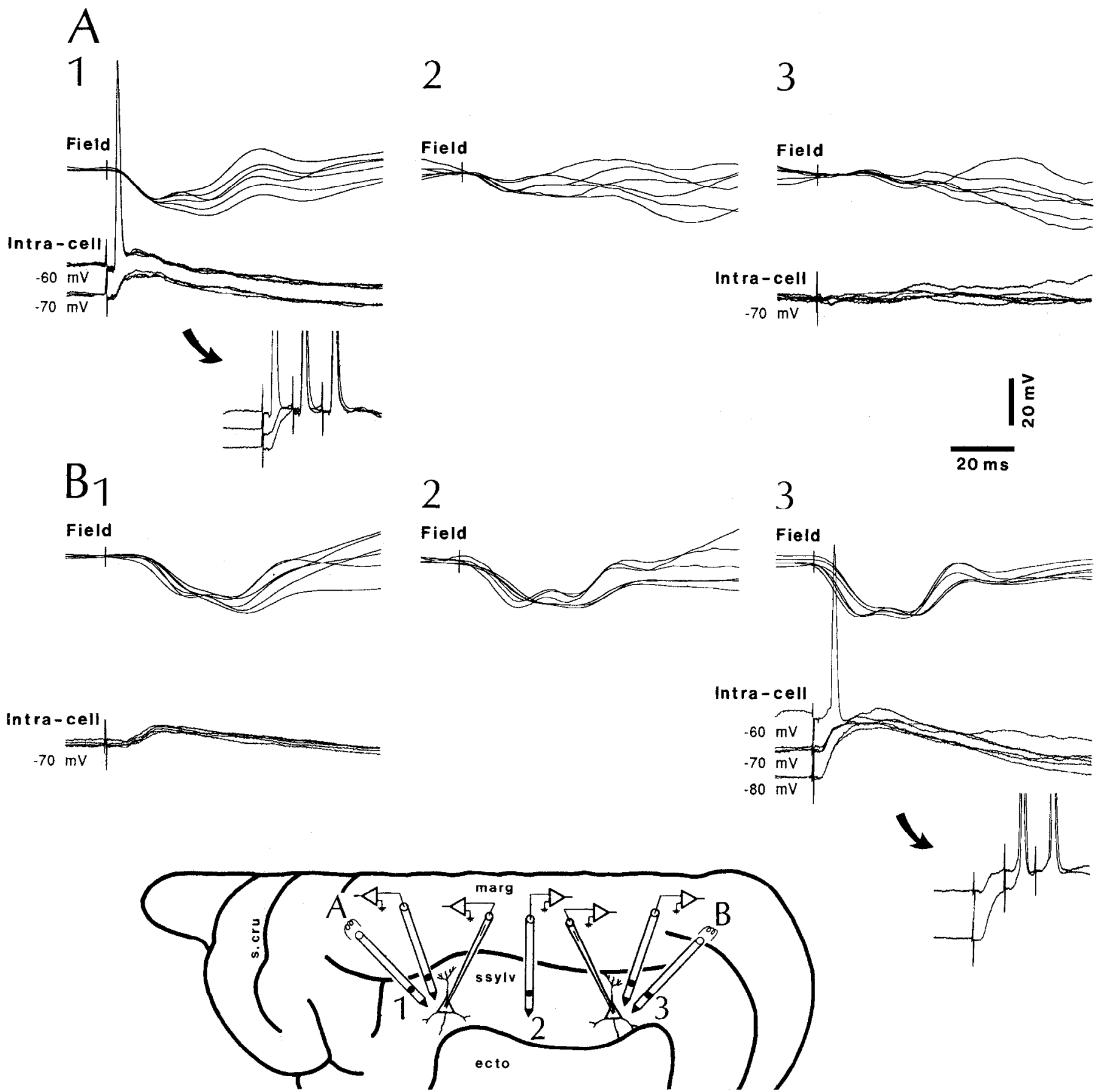

Figure 2. Electrophysiological identification of intracortical suprasylvian pathways. Two simultaneoủs intracellular recordings at sites $I$ and 3 , in the rostral and caudal suprasylvian gyrus (see bottom scheme). Close $(\approx 1 \mathrm{~mm}$ ) to each intracellular pipette there were two coaxial electrodes, one for bipolar recording of field potentials (FPs), the other for stimulation. An additional FP was recorded in the middle suprasylvian gyrus (2). Stimulation through the anterior electrode $(A)$ elicited in the closely recorded cell $(A I)$ an antidromic action potential, with a latency of 3 msec, at the resting membrane potential $(-60 \mathrm{mV})$. An EPSP, was revealed by hyperpolarizing the membrane at $-70 \mathrm{mV}$. The same stimulation evoked a biphasic depth-negative FP. The inset in Al shows three responses of this neuron, at different membrane potentials, to a three-shock train at 100 Hz. The anterior stimulation was less effective towards the caudal recording sites as shown by the progressively reduced amplitude of FPs and absence of overt EPSPs in the posterior intracellular recording ( $A 2$ and $A 3$ ). By contrast, stimuli delivered at the posterior site (B), induced EPSPS in both cells, with shorter latencies in the posterior neurons. The inset in B3 displays the temporal summation of EPSPs to $100 \mathrm{~Hz}$ stimulation.

to four amplitudes were observed as if they were triggered at different hot spots; and (2) it was a stable recording, lasting for $1 \mathrm{hr}$, and during all this period the cell oscillated and constantly responded to cortical stimulation. The incremental response elicited by three shocks is superimposed on a sweep triggered by a single volley. Both traces represent, respectively, the 20 th in a sequence of stimuli in which single shocks or pulse-trains were delivered at a rate of $0.5 \mathrm{~Hz}$. It is shown that the response to a first shock in a pulse-train already contains an incremental component, as compared to the response to a single shock. 


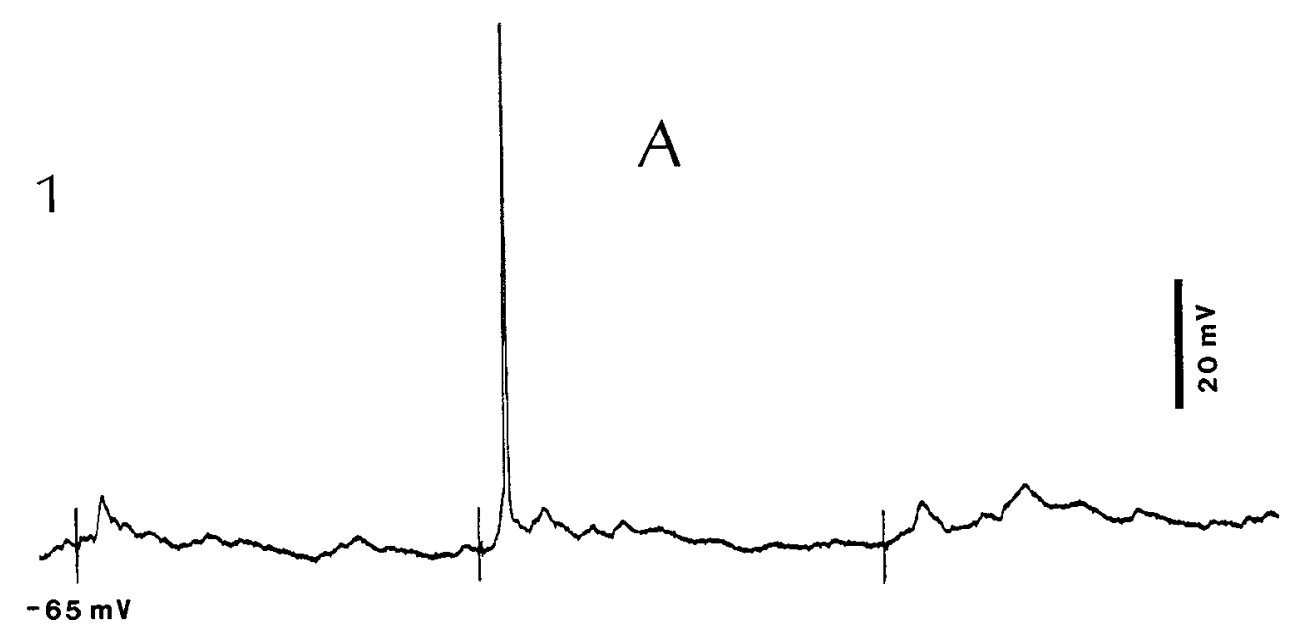

Figure 3. Augmenting responses elicited in intracellular recordings from the anterior suprasylvian gyrus by stimulating the posterior suprasylvian gyrus. Same arrangement of electrodes as in Figure 2. $\lambda l$, Responses to three stim uli at $10 \mathrm{~Hz}$. After the initial EPSP, a late excitatory component increases when evoked by the 2nd and 3rd stintuli. $A 2$, Average $(A V G)$ of 15 individual responses. The decaying slope of the response induced by the first stimulus of the train (from the top of the EPSP to the next shock; open arrow) is superimposed on the subsequent responses (dark arrow). The surface in black represents the contribution of the augmenting response. $B$, a different cell, depicts a presumed intradendritic recording (see text). Two sweeps are superimposed: the response to a three shock train at $100 \mathrm{~Hz}$ showing the augmenting responses to the pulse-lrairl, and the response to a single shock. Both sweeps were chosen after 20 pulse-trains or single shocks, delivered at a rate of $0.5 \mathrm{~Hz}$, and show that the response to a first shock in the train already contains an incremental component as compared to the response evoked by a single shock.

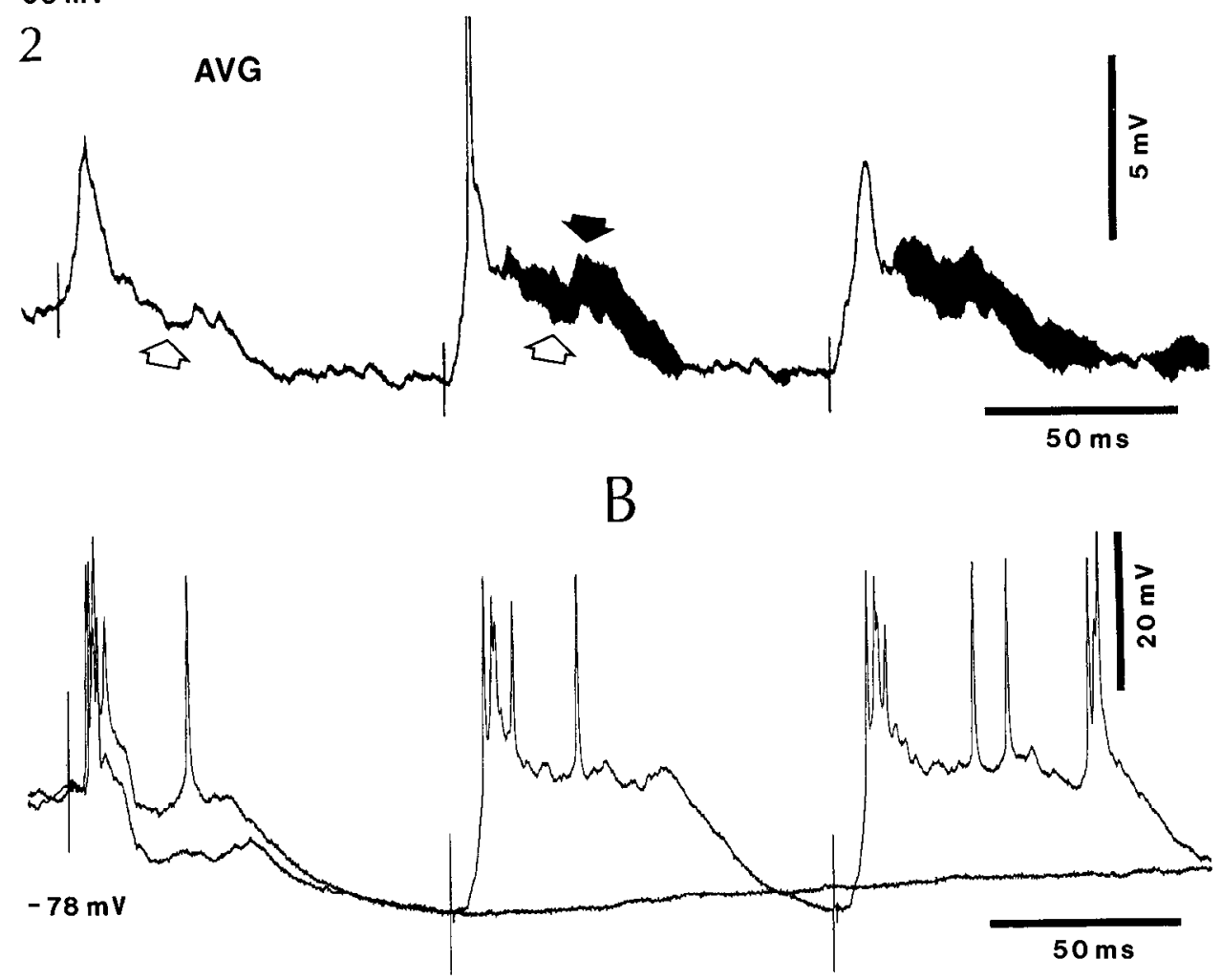

Lidocaine infusion in the middle suprasylvian gyrus, between the posterior stimulating and the anterior recording sites (see site 2 in the scheme of Fig. 2), disrupted the intrasuprasylvian synaptic linkage (Fig. 4). During the control period of a double intracellular recording, both posterior and anterior neurons were driven orthodromically, and the adjacent FPs reflected the excitation with depth negativities (Fig. 4, left). After injection of lidocaine $(2 \mu \mathrm{l}, 20 \%$ ), the responses in the closely located, middle suprasylvian lead (Mid.), and beyond it, in the anterior (Ant.) suprasylvian gyrus, were blocked (right). The intracellular and FP responses close to the posterior stimulating electrode (Post.) were not affected.

The functional inactivation produced by lidocaine infusion interrupted reciprocal, anterior-to-posterior and vice versa, suprasylvian responses (Fig. 5). Each sweep in this figure, with the exception of the two insets at the left, derives from an average of 40 intracellular and FP responses. The responses at the siles close to the stimulating electrode (Ant. in Fig. $5 A$ and Post. in
Fig. $5 B$; see again the arrangement of stimulating and recording electrodes in Fig. 2) were unaffected by the injection of lidocaine in the middle suprasylvian gyrus. The amplitude of the FP response close to the injection site (Mid.) was strongly reduced, while the intracellular and FP responses at the sites opposite to those of stimulation were completely blocked.

The augmenting component was affected by intracortical inactivation. Before the lidocaine injection between the posterior stimulating and anterior recording electrodes (Fig. 6, Control), five shocks at $10 \mathrm{~Hz}$ clicited an incremental response (black surface) that is shown by superimposing the response to the first shock (open arrow) over each response to successive stimuli (solid arrow). The early, fast excitatory component did not change or even decreased at stimuli subsequent to the first one (compare the response to a single shock with the response to the first shock in a pulse-train). A similar decrease in the early response, sinultaneously with a selective increase in the late excitatory responses during augmenting responses, have previously 


\section{Control}

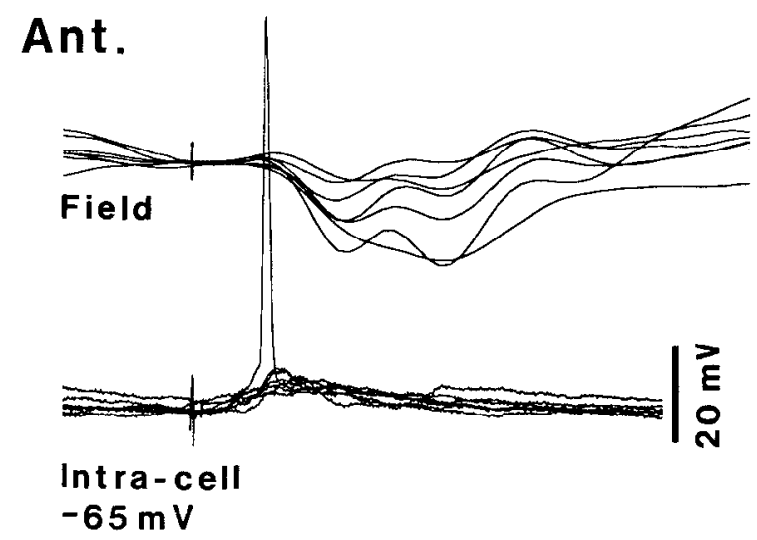

Mid.

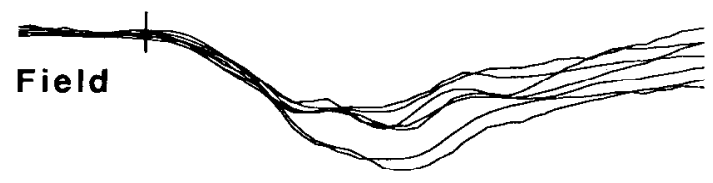

Post.
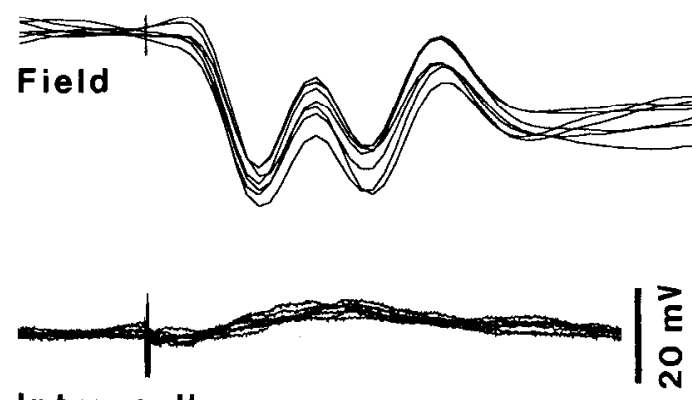

Intra-cell

$-80 \mathrm{mV}$

\section{After lidocaine}
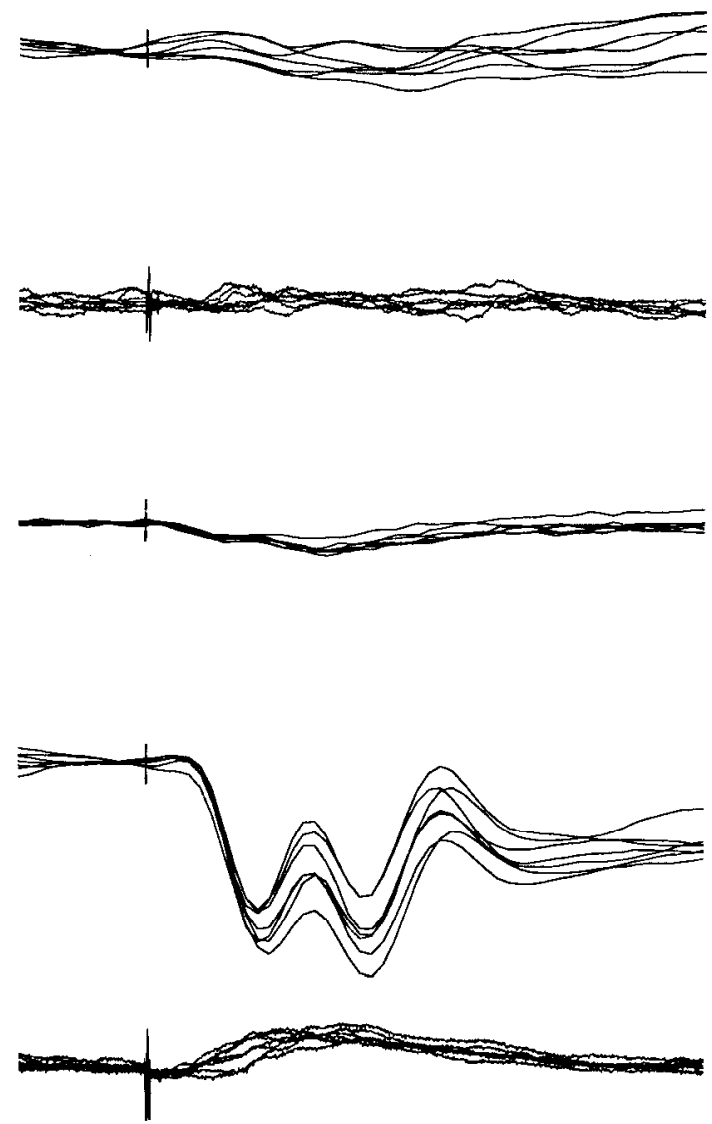

$10 \mathrm{~ms}$

Figure 4. Effect of lidocaine inactivation on intracortical responsiveness. Superimposed sweeps from a double intracellular recording in anterior and posterior sites of suprasylvian gyrus. Stimulation was applied close to the posteriorly located neuron (similar arrangement of electrodes as in the scheme shown in Fig. 2). During the control period (left), both cells were orthodromically driven and the respective FP recording displayed depth-negative components. After lidocaine infusion in the middle suprasylvian gyrus (Mid.), at half distance between the anterior (Ant.) and posterior (Post.) recordings, the response in the anterior cell and FP, as well as in the FP close to the injection site, were abolished, while the responses in the posterior site preserved the same pattern (right).

been shown in extracellular recordings from primary somatosensory and parietal association cortices (see Fig. 5 in Steriade, 1978). Calculating the black surface after each stimulus, and dividing it by the corresponding surface of the control response to the first shock, it results that the increment from the first to the second shock is $100 \%$, from the second to the third $125 \%$, from the third to the fourth $155 \%$, and from the fourth to the last $184 \%$. This indicates a continuous progression of $25-30 \%$. Four minutes after lidocaine infusion (Fig. 6, bottom), the amplitude of the synaptic response to the first stimulus was diminished by about $30 \%$ and the latency of the EPSP increased from 3 to $8 \mathrm{msec}$. The same procedure of superimposing the response to the first stimulus (open arrow) over the following responses (solid arrow) was applied again. It results that the early excitatory response was diminished by the amount indicated by the dotted surface, while later components still exhibited an incremental response.

\section{Effects of functional intracortical disconnection on the} synchronization of the slow oscillation

The effect of lidocaine infusion was first investigated on the slow oscillation of FPs. Five bipolar FPs recordings within the suprasylvian gyrus, between the anterior stereotaxic plane 15 (electrode 1) and plane 0 (electrode 5), are depicted in the top figurine of Figure 7 . The injection site, marked by arrow, is at plane 7 , between the rostral and caudal recording sites. During the control period all leads displayed a synchronous slow oscillation at about $0.7 \mathrm{~Hz}$ (the underlined sequences in left panels are expanded at right). The inactivation of lead $2(1 \mathrm{~mm}$ caudally from the tip of the cannula) began towards the end of the intracortical infusion of lidocaine $(5 \mu \mathrm{l}, 40 \%$ ) (see open bar at left in the second panel). The right detail from the underlined period marks the desynchrony between anterior and posterior sites, although each of them continued to display the slow oscillation and despite the fact that the synchrony among the three posterior 


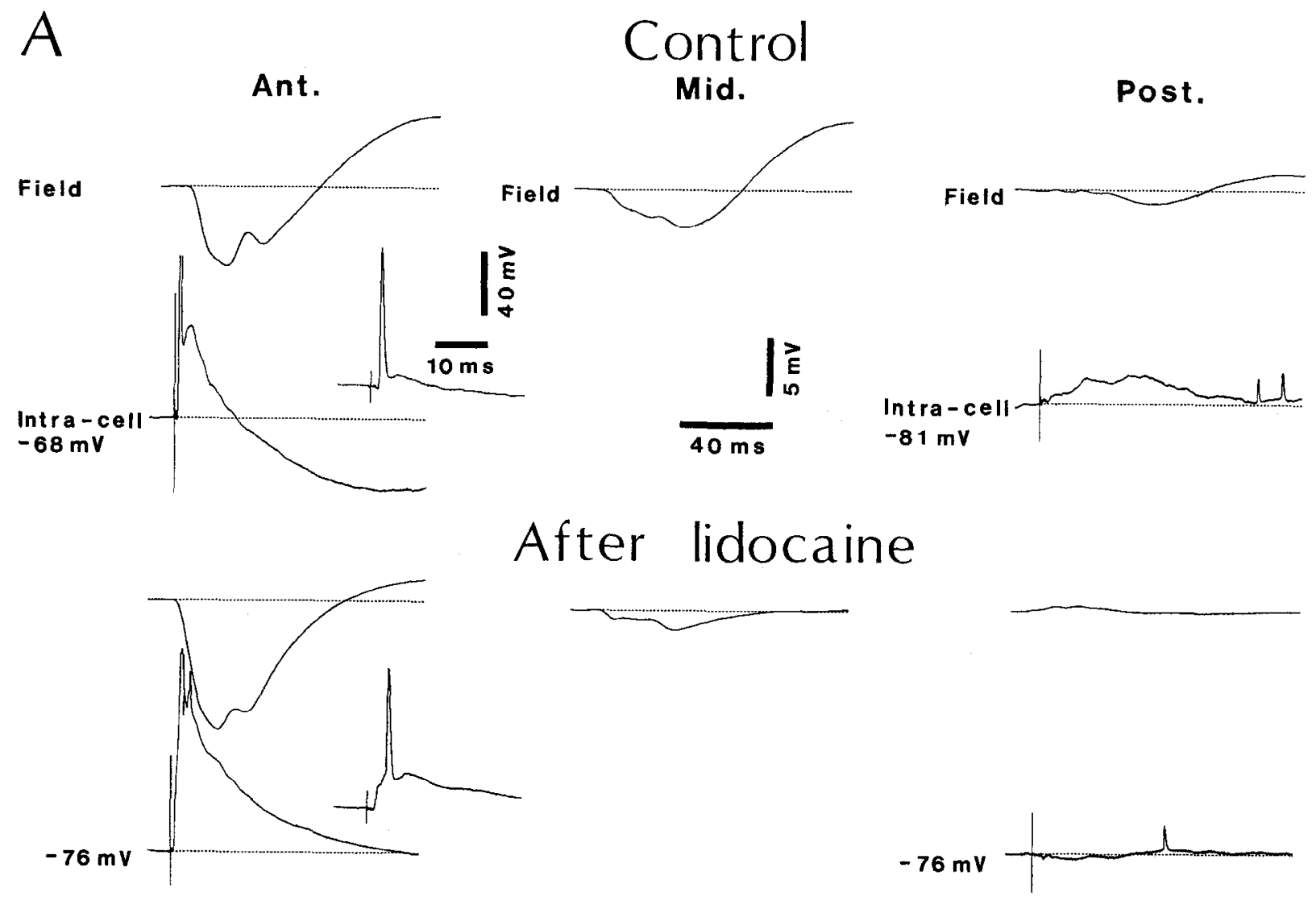

B

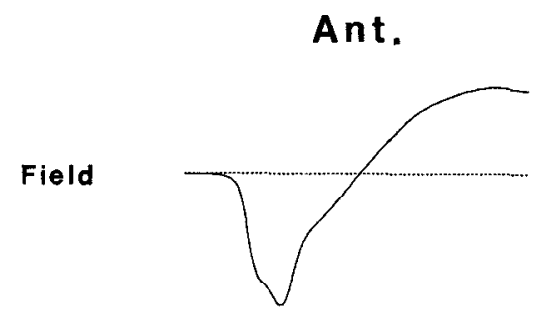

Intra-cell
$-70 \mathrm{mV}$
Control

Mid.

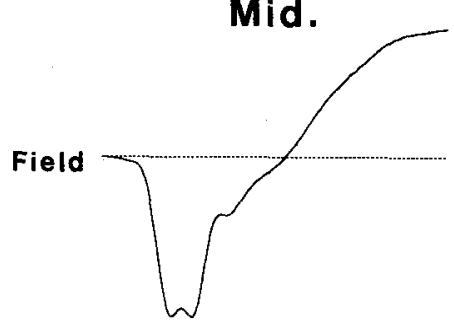

Post.

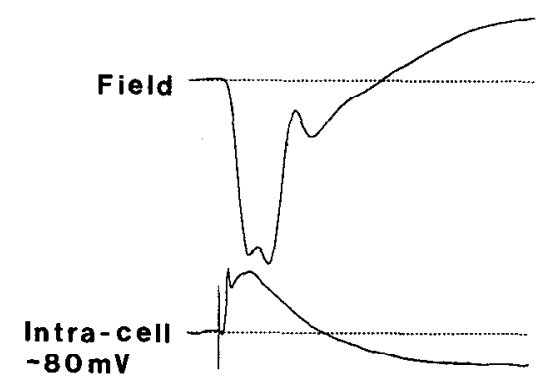

\section{After lidocaine}
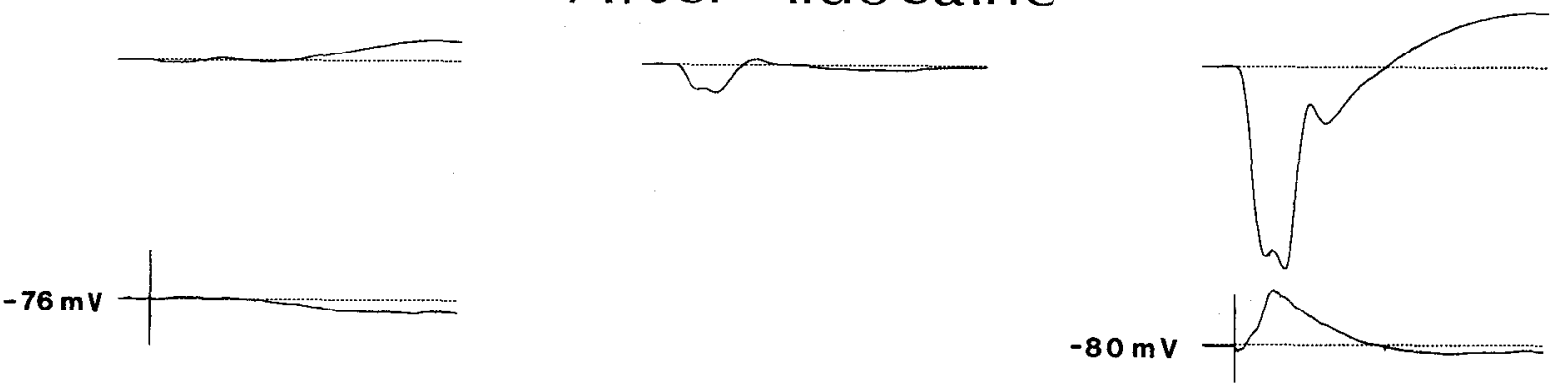

Figure 5. Functional disconnection of anterior (Ant.) and posterior (Post.) suprasylvian foci by lidocaine inactivation within the middle (Mid.) suprasylvian territory. Averaged evoked potentials $(n=40)$ in a double intracellular tecording from anterior and posterior suprasylvian sites. A, Anterior stimulation. The control period shows antidromic activation of the anterior neuron (see inset), orthodromic response of the posterior one, 

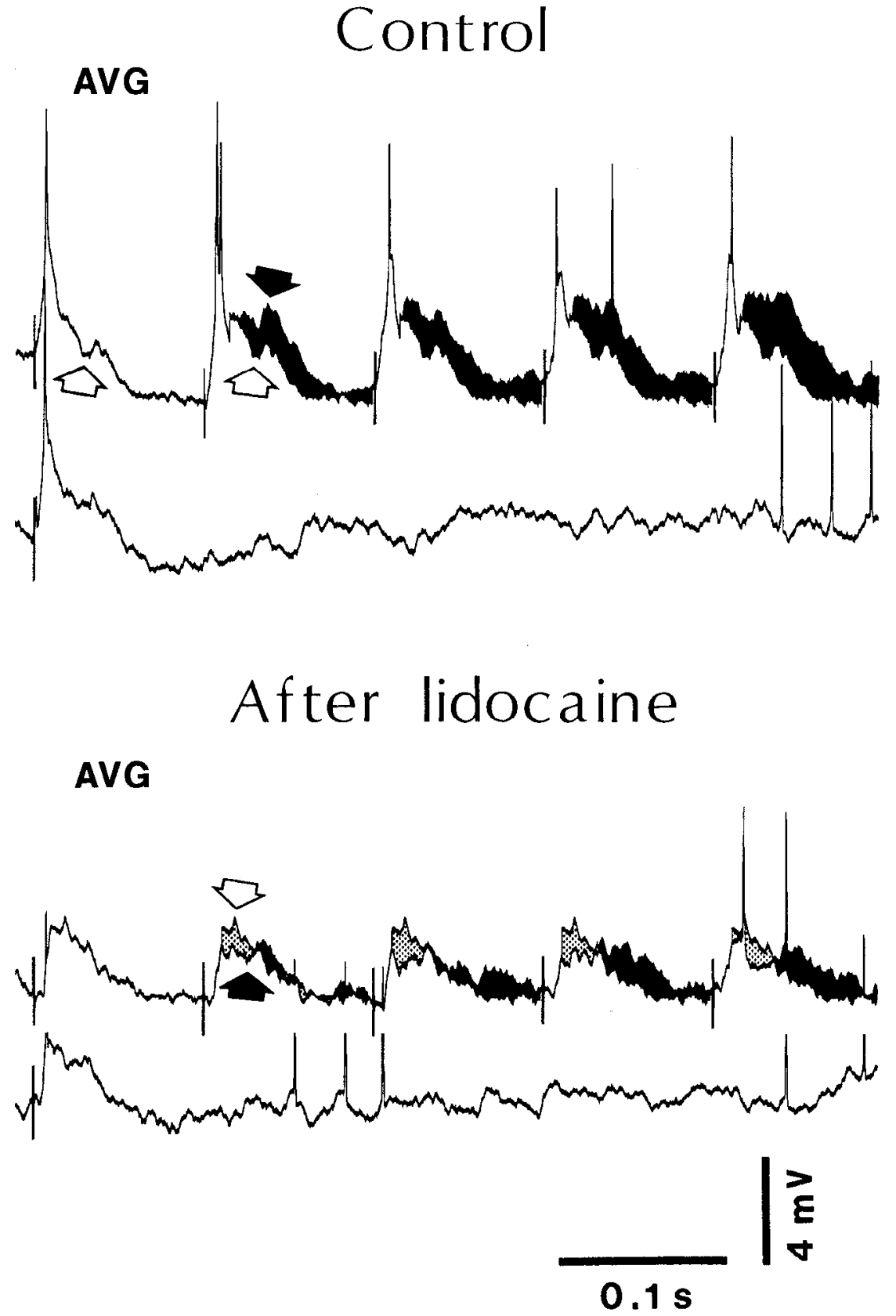

Figure 6. Effect of intracortical lidocaine inactivation on the augmenting response. Anterior suprasylvian intracellular recording and posterior suprasylvian stimulation with five shocks at $10 \mathrm{~Hz}$ (upper traces) and with single shock (lower traces). Averages ( $A V G$, $n=15$ ) of incremental responses (black surface) shown by superimposing the response to the first stimulus (open arrow) over each subsequent response (solid arrow). During the Control period incrementing responses are observed after the second stimulus; see upper sweep in which the response to the first shock in the train of five stimuli is superimposed (open arrow) on the subsequent responses (solid arrow), leaving in black the augmenting surface. Four minutes after injecting lidocaine in a cortical area between the recording and stimulating sites, the amplitude of the evoked response diminished as compared to the control situation (see single-shock stimulation). With the five shock train, superposition of the first response over the other ones (open arrow) disclosed two features: diminution of the first component (dotted surface) and preservation of a reduced incrementing component (in black). leads ( 3 to 5 ) was preserved. Four minutes after the end of the injection, the inactivation spread to channel 3 , as indicated by its reduced amplitude. At this time, the rostral and caudal recordings oscillated independently and few signs of synchrony survived between them (see detail expanded at right). The posterior leads ( 4 and 5 ), not affected by lidocaine, remained synchronized. Four hours after lidocaine injection, both leads 2 and 3 recovered their normal activity (as shown by the amplitude and general aspect of FPs) and the synchrony between all channels was recovered.
The sequential field correlation analysis of data depicted in Figure 7 is shown in Figure 8. The state of synchrony between channels 1, 4, and 5 (see cortex figurine in the middle of figure) was assessed by performing sequential correlations of the type described in Materials and Methods. Channels 2 and 3 were not analyzed because of their transient inactivation (sce pancls during lidocaine injection and $4 \mathrm{~min}$ after in Fig. 7). Each panel depicts 3-D surfaces and contour maps for both cross-correlations between anterior-posterior sites (CROSS 1-4) and between posterior leads (CROSS 4-5). The control synchrony was char-

$\leftarrow$

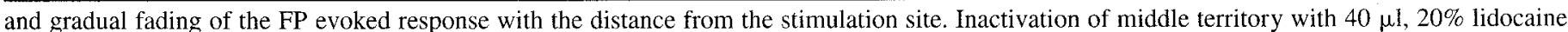

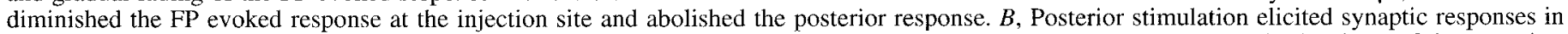

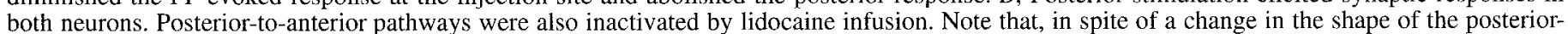
cell EPSP, the pattern of the posterior FP remained unchanged. 


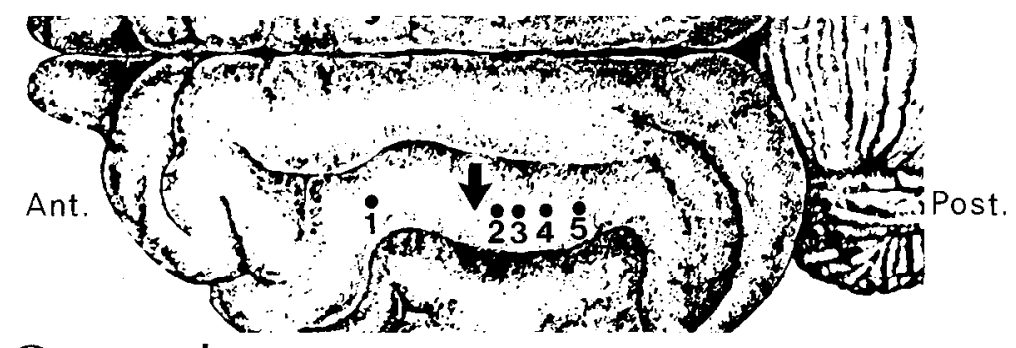

\section{Control}

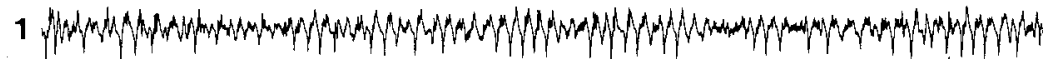

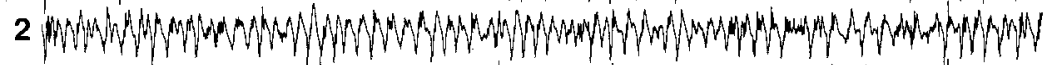

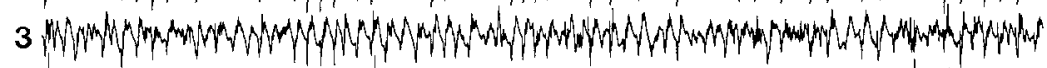

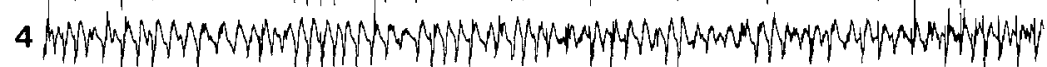

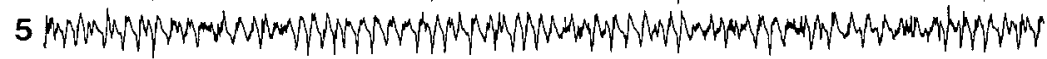

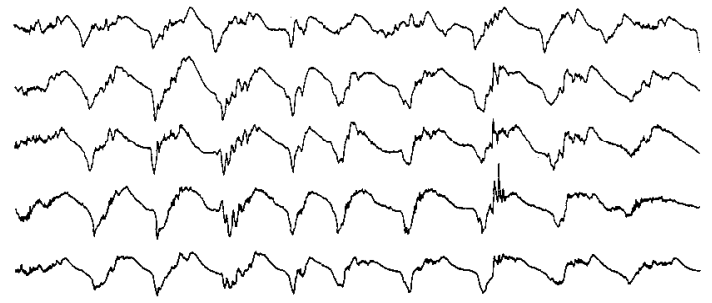

\section{Lidocaine}

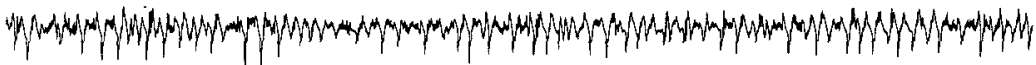

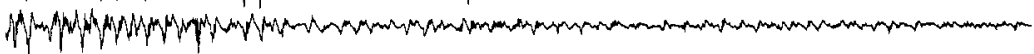

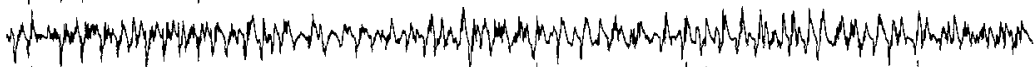

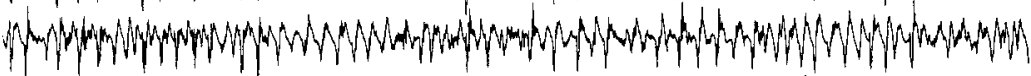

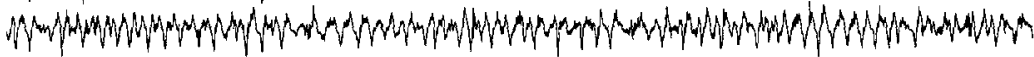

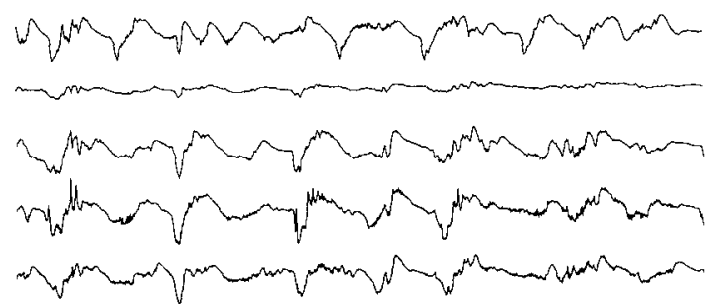

LIDOCAINE

4 min after

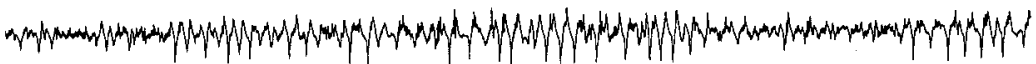

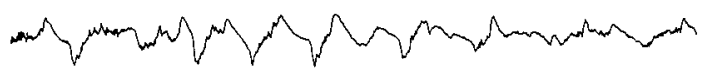

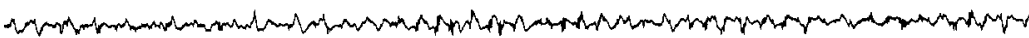

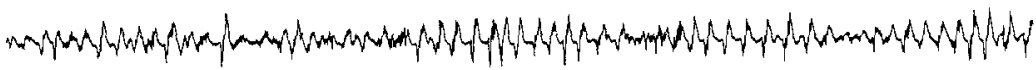

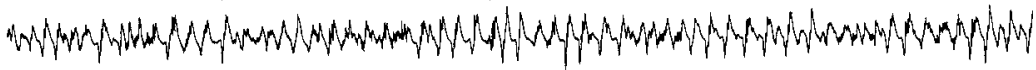

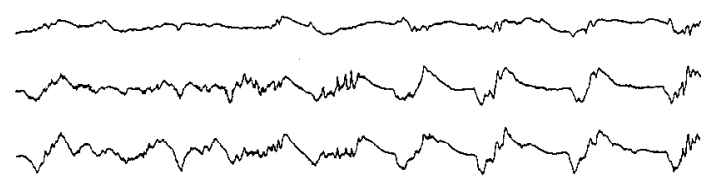

\section{4 hrs after}

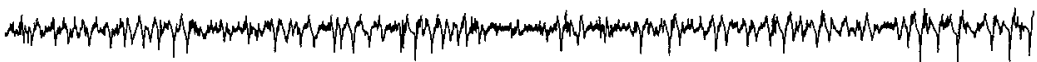

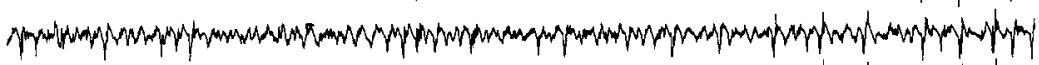

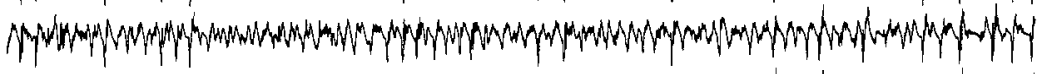

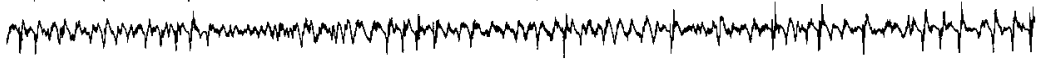

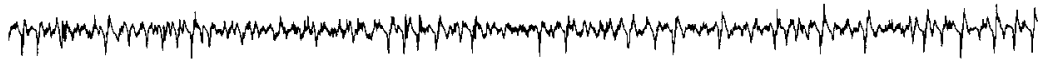

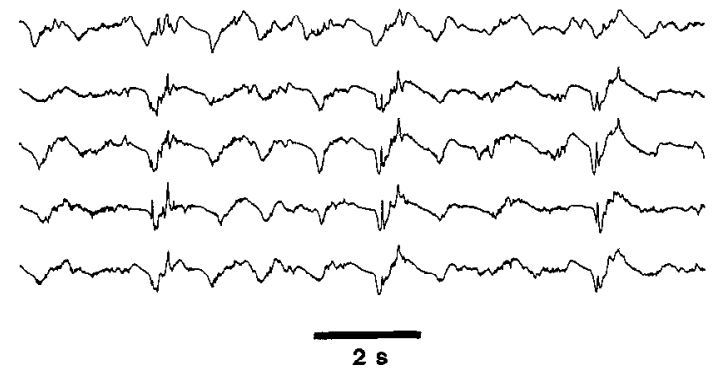

$10 \mathrm{~s}$

28

Figure 7. Effect of lidocaine injection on FPs synchrony within the slow oscillation. Five bipolar electrodes field recordings in the suprasylvian gyrus (see figurine) between anterior 15 (electrode 1) and anterior 0 (electrode 5). Injection site marked by arrow is at anterior 8 . During the control period all leads display a synchronous slow oscillation of $\approx 0.7 \mathrm{~Hz}$ (see underlined sequence expanded at right). Immediately after intracortical injection of lidocaine $(5 \mu \mathrm{l}, 40 \%$, during open bar at left of the second panel), inactivation of electrical activity was seen on lead 2 ( $1 \mathrm{~mm}$ caudally from the tip of the cannula). The detail from the underlined period shows an epoch of desynchrony between anterior and posterior sites, while all of them continued to express the slow oscillation. Synchrony between posterior leads was preserved. Four minutes after the end of the injection, the inactivation spread to the neighboring channel 3 (as indicated by reduced amplitude of electrical activity), while rostral and caudal recordings oscillated independently (see also detail at right). Four hours after the injection synchrony between all channels was restored. In this and similar figures, polarity in FPs recordings is similar as in intracellular recordings (positivity up). 

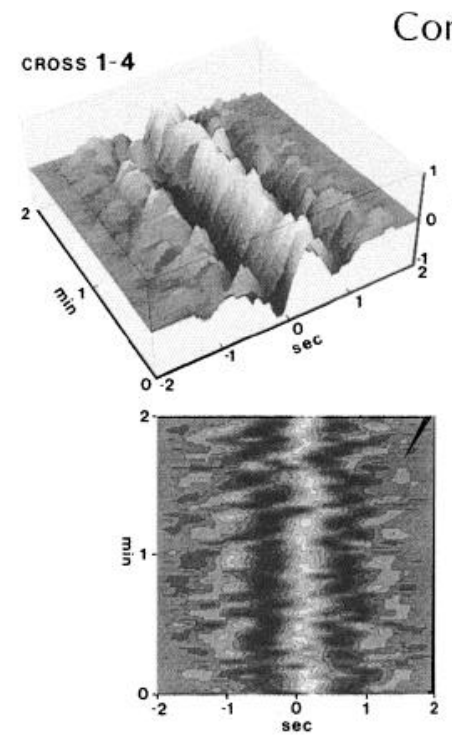

4 min after
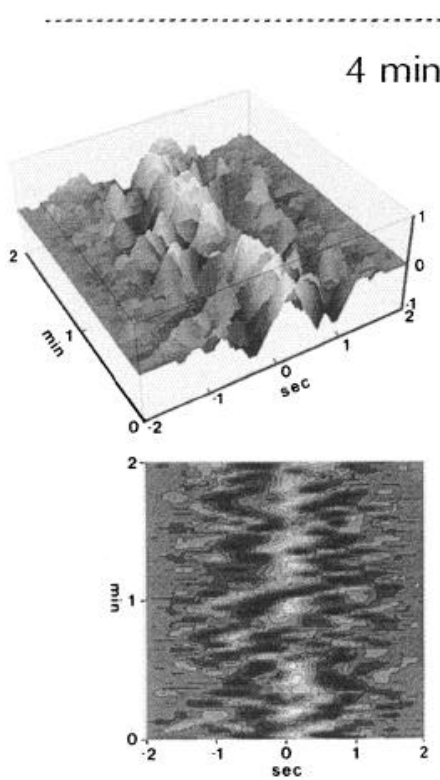

Control
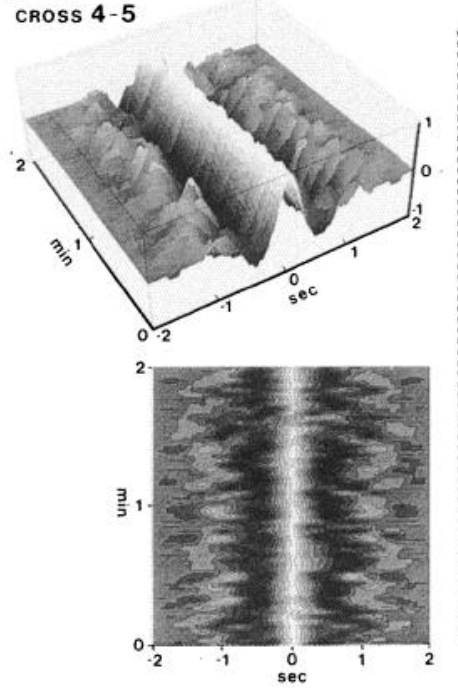

\section{Lidocaine}
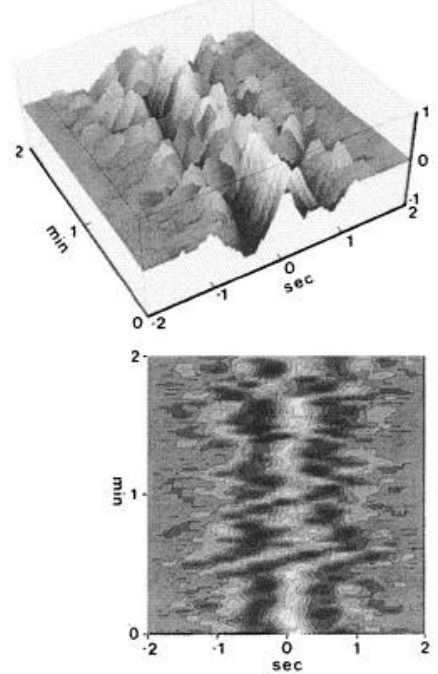
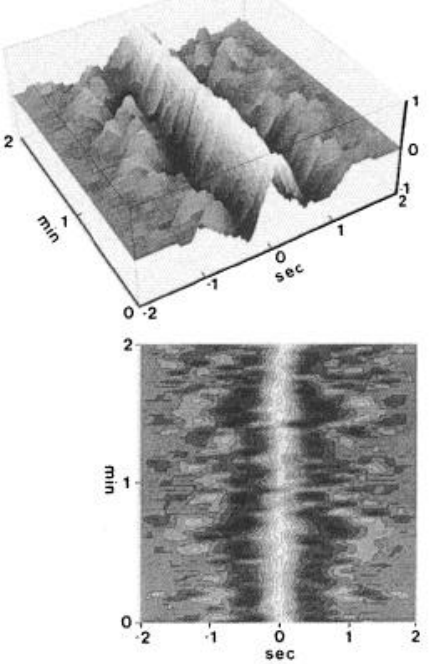
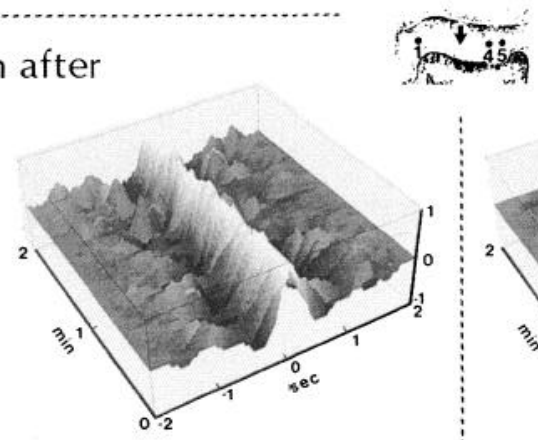

4 hrs after
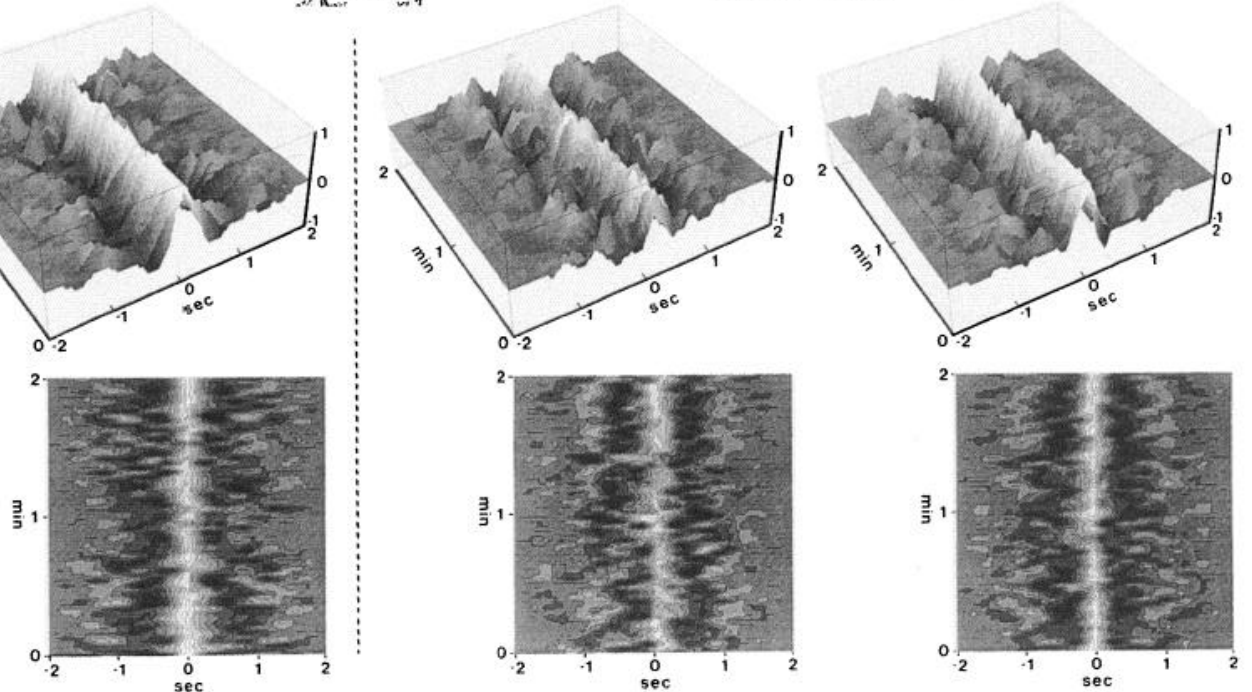

Figure 8. Sequential field correlation analysis of data presented in Figure 7. Periods of 2 min, including the ones presented in Figure 7, were analyzed. See text for details and full explanation.

acterized by well aligned, high central peaks, and was more powerful between close foci (4-5) than between more distant ones (1-4). The synchrony coefficients (SyCo) were 0.88 and 0.55 , respectively. As soon as lidocaine inactivation became effective in channel 2 (see second panel in Fig. 7), the previous pattern of anterior-posterior (CROSS 1-4) synchrony was replaced by a blurred sequence of lower peaks or lower valleys deviating from the central plane that was a landmark of synchrony. During that period, SyCo dropped to 0.34 . The appearance of correlation peaks at random latencies was due to the survival of the slow oscillation, with the same features of waves, on both sides of the inactivated middle area of the suprasylvian gyrus. The dispersion of the peaks and their random occurrence was the consequence of a loss of links between the two oscillatory pools. As the inactivation spread (4 min after lidocaine injection), the randomness of correlation peaks progressed and time-lags of $\pm 1 \mathrm{sec}$ appeared. However, the SyCo stabilized at 0.34. During the last two periods (lidocaine and $4 \mathrm{~min}$ after), the synchrony between posterior foci and the respective SyCo remained unaffected. Four hours after the lidocaine injection, when the recovery of electrical activity was observed in the previously inactivated areas, sequential correlations recovered their initial aspect and SyCo became 0.5.

The effects of reversible inactivation on SyCo was calculated in a series of recordings and the quantitative results on the drop in synchrony, which were found to be similar to those obtained after transections, are reported at the end of the following section (Effects of intracortical transections ....).

It should be stressed that the disruption of the intracortical synchrony did not abolish the oscillation in distantly located cortical areas, but it produced a change in the frequencies of the slow rhythm. This is shown in Figure 9, with FPs recordings from the anterior and posterior suprasylvian gyrus (area 5 and area 21 , respectively), before and after lidocaine inactivation as well as during the recovery of synchrony between the two sites. The bottom panels in Figure 9 illustrate the commonalities 

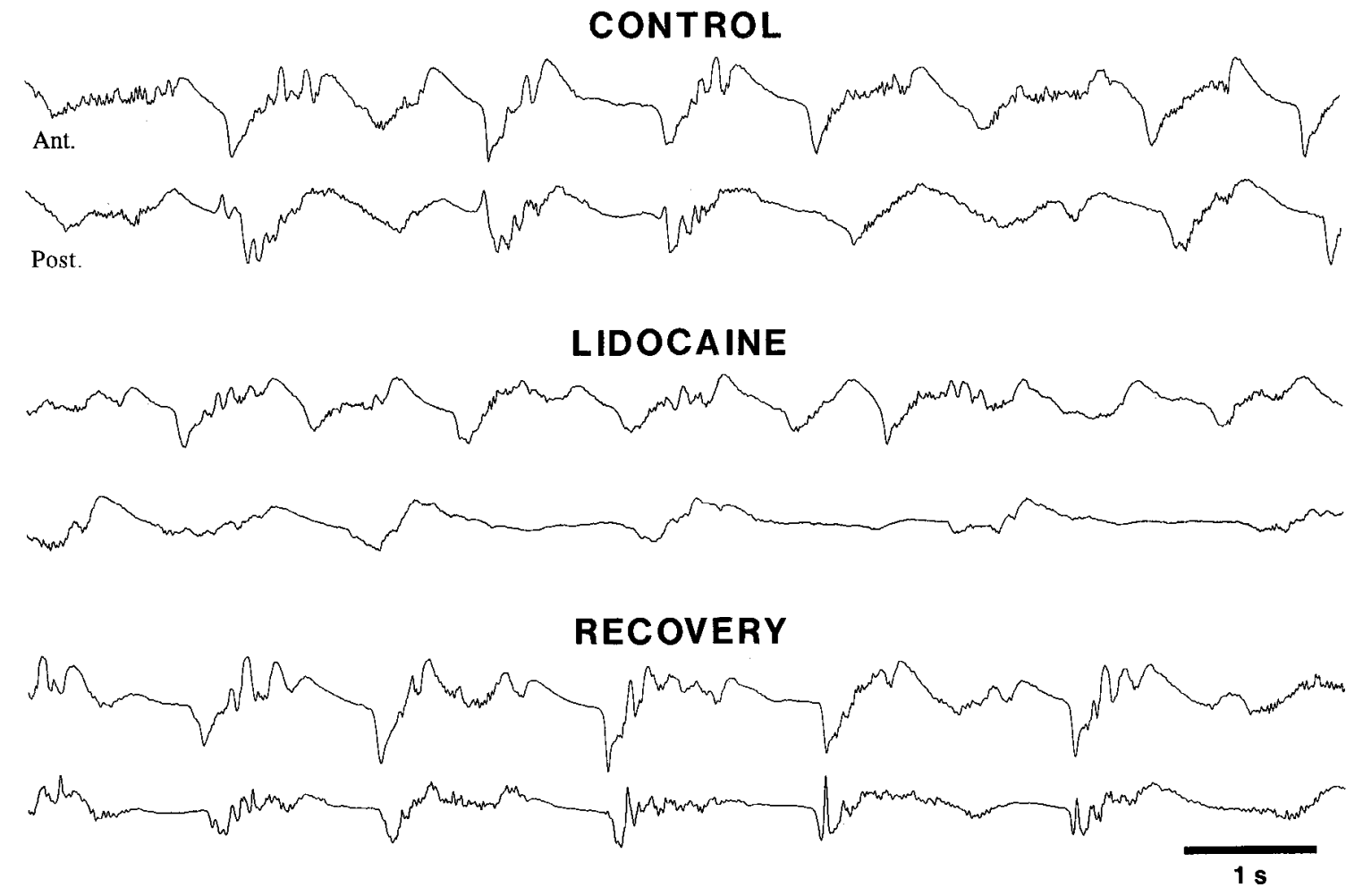

\section{CONTROL}
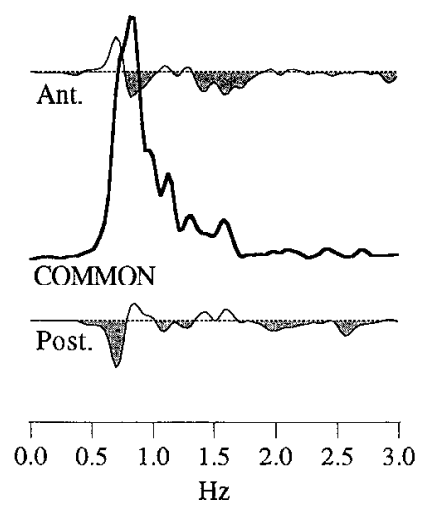

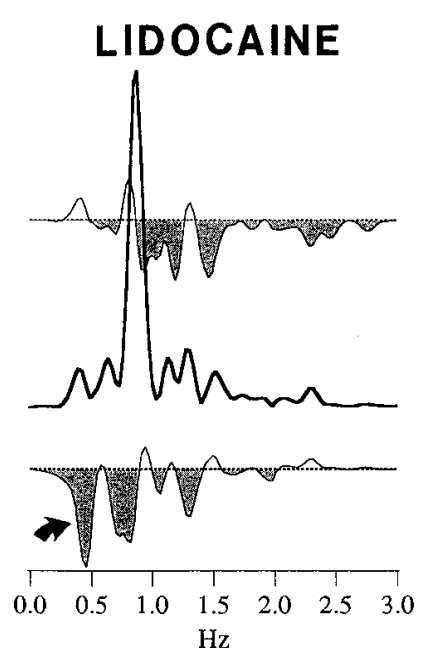

Figure 9. Disruption of intracortical synchrony does not abolish the oscillatory propensity of disconnected foci, but reveals changes in the frequencies of their rhythms. Slow oscillation of FPs recorded from anterior (Ant.) and posterior (Post.) parts of the suprasylvian gyrus, before, after lidocaine inactivation in the middle suprasylvian gyrus, and during recovery ( $3 \mathrm{hr}$ after lidocaine infusion). Note disruption of synchrony between Ant. and Post. sites after lidocaine, and lower frequency of the slow oscillation at the Post. site. The bottom panels illustrate the FFT analysis of the FPs during the three periods (see Data analysis in Materials and Methods). The shadowed areas in the differential FFTs (arrow in the IIDOCAINE panel) indicate the independent activities in the two oscillatory pools and their significant increase after lidocaine-induced decoupling. For further explanation, see text.

(COMMON in middle rows) and differences (shadowed parts in the upper and lower rows) between the frequencies observed in the two channels during control and recovery periods, as opposed to the effects during lidocaine inactivation (see Data analysis in Materials and Methods for details on auto- and crossspectra). In the experiment illustrated in Figure 9, the coherent slow oscillation had a frequency of $0.7-0.8 \mathrm{~Hz}$ (see main peak of the cross-spectrum, COMMON) while the excess surface left out of the $0-3 \mathrm{~Hz}$ frequency band represented less than $10 \%$ of the total spectrum. Two independent oscillators constituted the common peak. Besides the common oscillatory frequency expressed by the cross-spectrum, the differential FFTs (shadowed parts in the upper and lower rows) revealed the tendency of the anterior area to oscillate at $0.8 \mathrm{~Hz}$, whereas the posterior part of the gyrus oscillated at a lower frequency, $0.7 \mathrm{~Hz}$. After lidocaine inactivation, the frequency differences between the two oscillat- 


\section{Control}

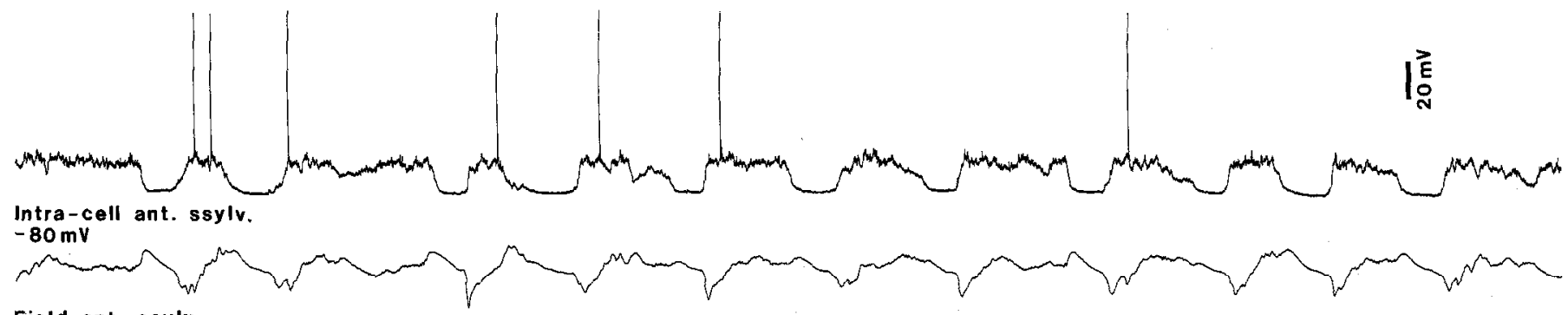

Field ant. ssylv.

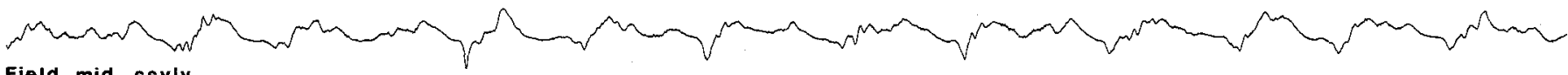

Field mid. ssyiv.

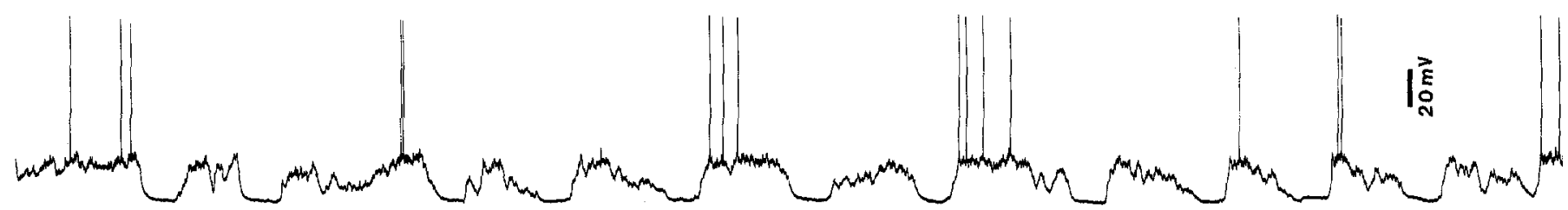

Intra-cell post ssylv.

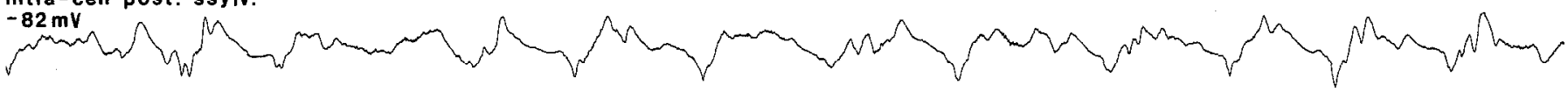

Field post. ssylv.

\section{After lidocaine}

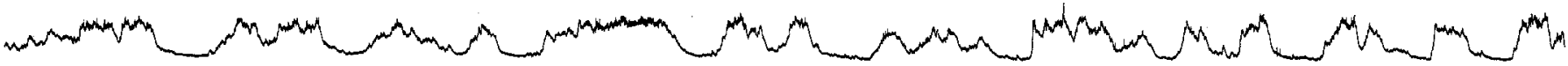

Intra-cell ant. ssylv.

(24 mV

Field ant, ssylv.

Field mid. ssylv.

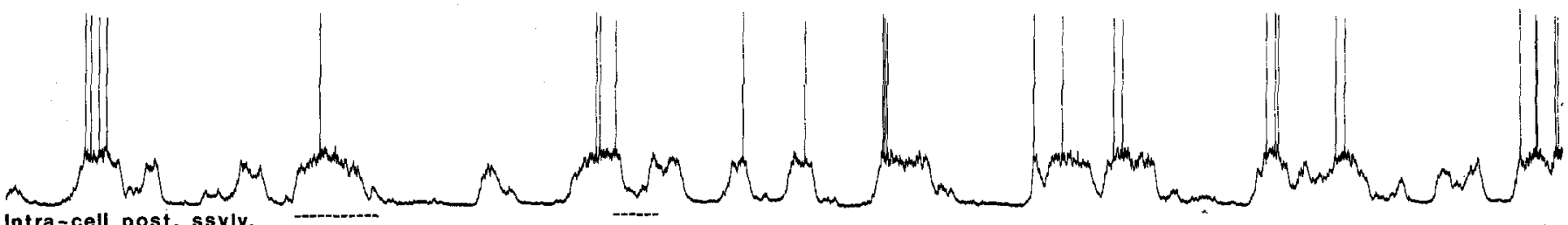
Intra-celi post. ssyiv.

Field post. ssylv.

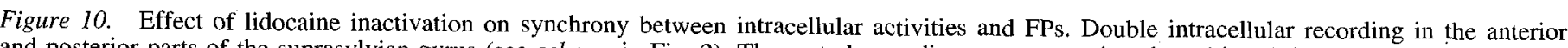

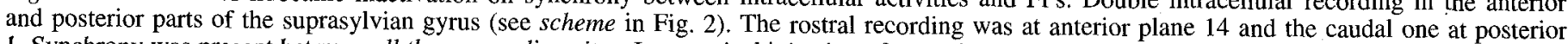

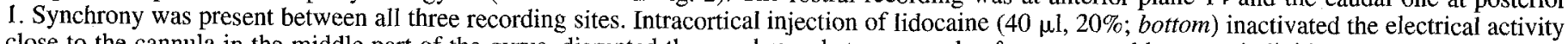

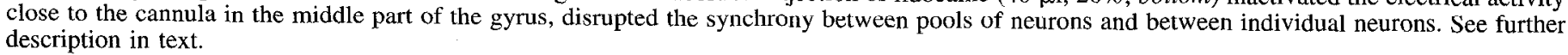


Control
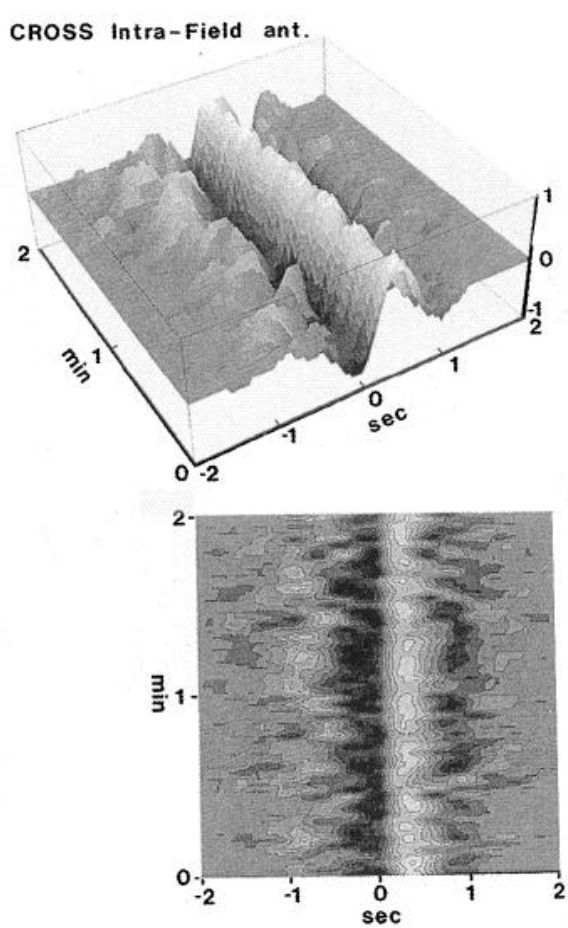

cROSS intra ant--post.
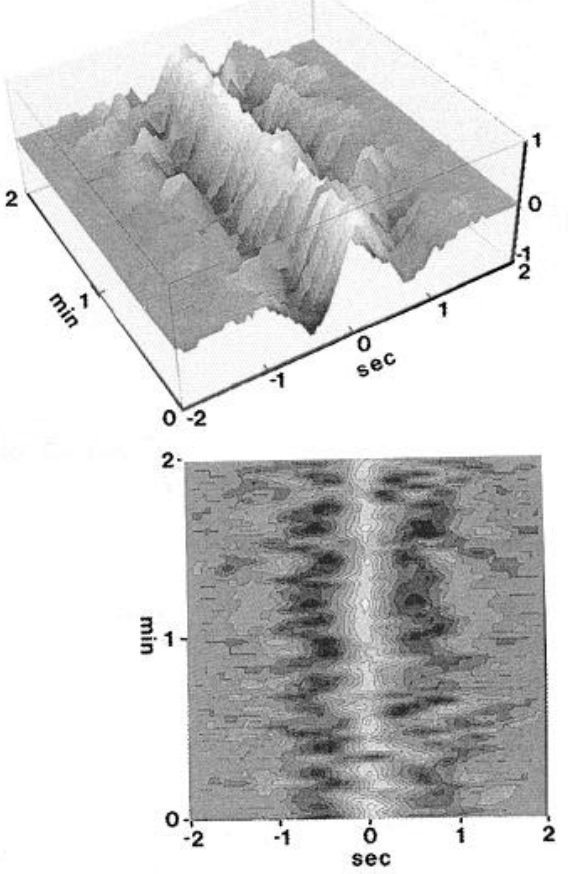

After lidocaine
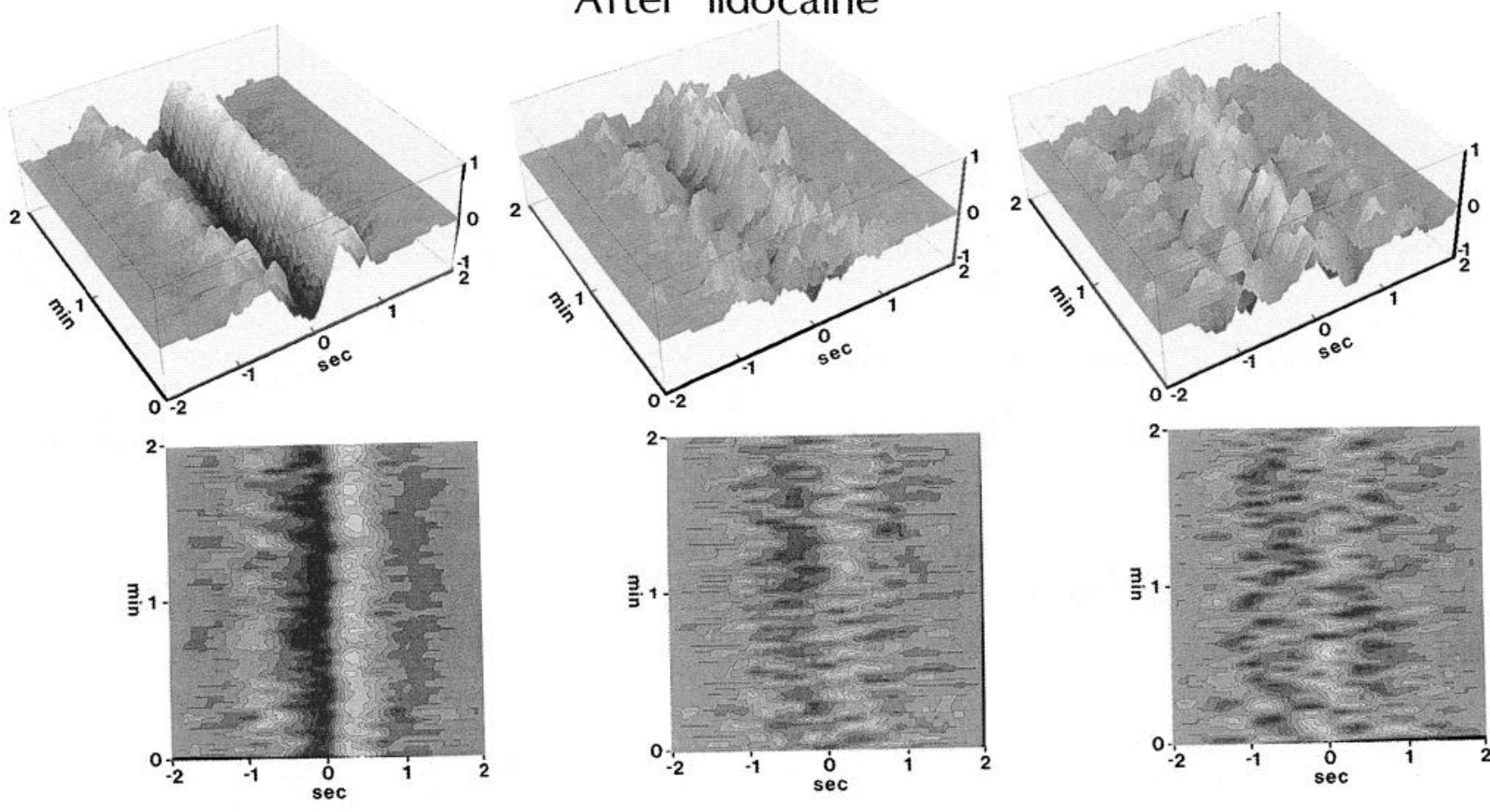

CROSS Field ant.-post.
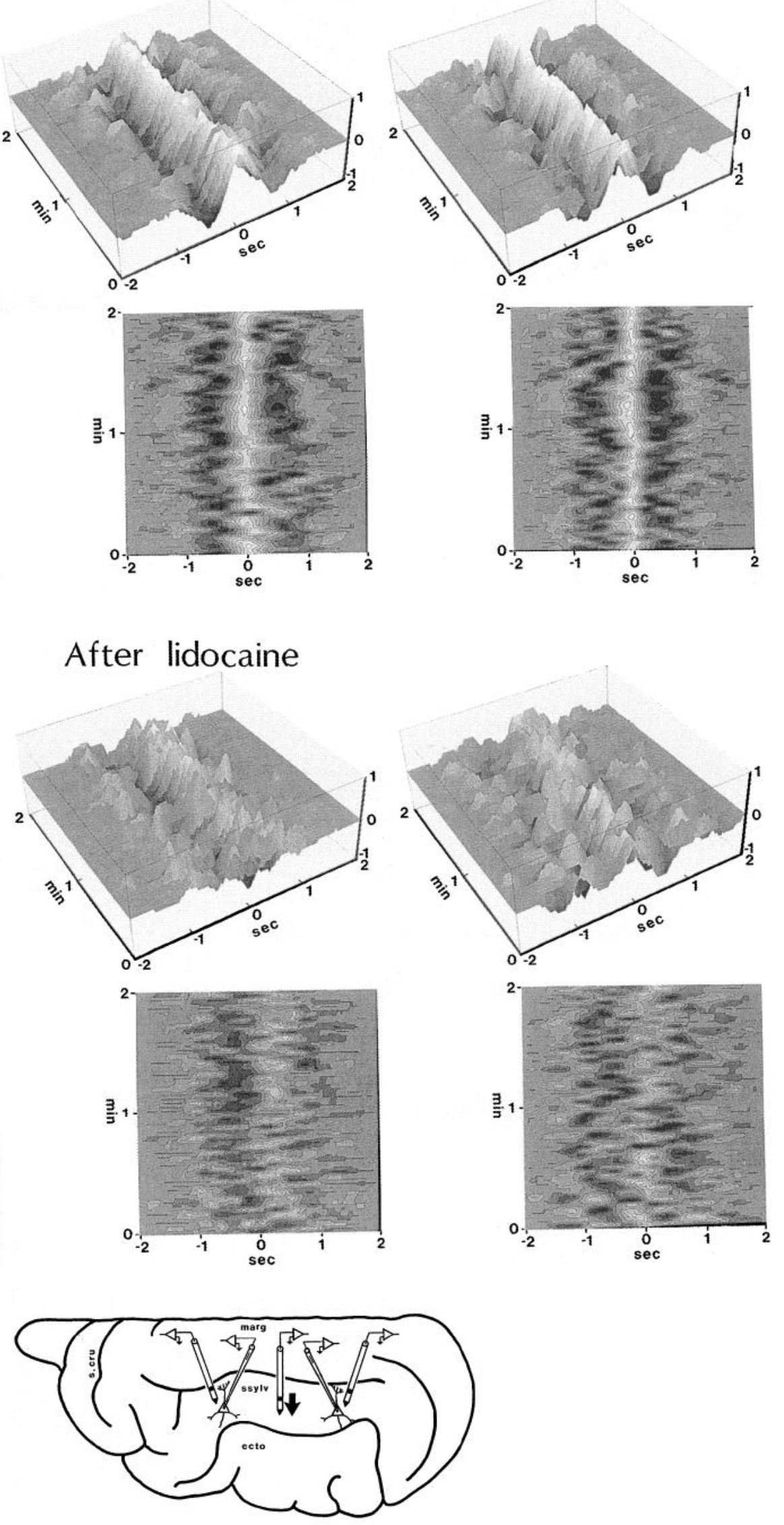

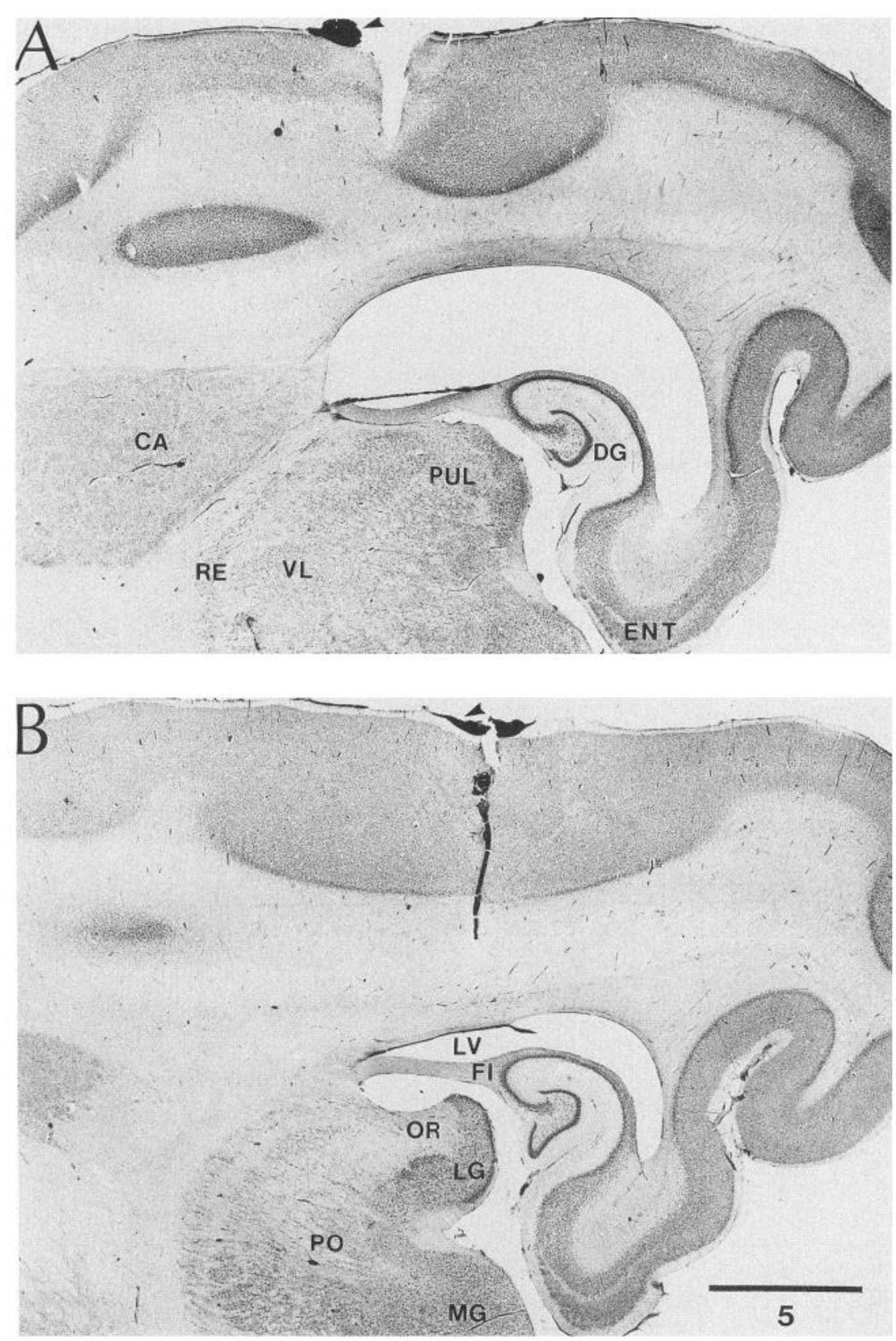

Figure 12. Histological aspects of intracortical transections in the middle of the suprasylvian gyrus. $A$ and $B$, Two experiments. Thionine-stained parasagittal sections. Arrowheads to the cortical surface point to the burned pia by silver nitrate. Horizontal bar in $B(\mathrm{~mm})$ is also valid for $A$. $C A$, Caudate nucleus; $D G$, dentate gyrus; $E N T$, entorhinal cortex; $F I$, fimbria; $L V$, lateral ventricle; $O R$, optic radiation; $P O, P U L, R E$, and $V L$, posterior group, pulvinar, reticular, and ventrolateral thalamic nuclei. ing suprasylvian foci became much more obvious due to their uncoupling, and a slower oscillation was revealed at about 0.4 $\mathrm{Hz}$ in the posterior recording (arrow in middle panel), as also evident from the rough data illustrated above. During the recovery period, $3 \mathrm{hr}$ after lidocaine infusion, the contribution of the two channels was similar to the control period.

We calculated the surface of the negative part of the differential spectrum from all 34 recording sessions (such as the shadowed parts in the bottom panels of Fig. 9). These data indicate that the increased surface from the control periods to the reversible inactivation periods was of $292 \pm 25 \%$, while the recovery was within $8 \%$ of the control periods. The threefold increase in the surface of independent oscillations matches the decrease by the same amount of the SyCo under the same experimental conditions (see below).

The effect of lidocaine inactivation on intracortical synchrony was also studied with double intracellular recordings together with their FPs within the anterior and posterior parts of the suprasylvian gyrus (Fig. 10) (see arrangement of electrodes in the scheme at the bottom of Fig. 11). The control period displays the rhythmic activity (at about $0.7 \mathrm{~Hz}$ ) of the two intracellularly recorded neurons and of the closely recorded FPs, and is also seen in the FPs recorded from the middle part of the gyrus. The onset of an intracellular depolarization corresponded to a depth negativity in the adjacent FP, while hyperpolarizations were reflected by depth positivities. Synchrony was present between all

Figure 11. Sequential correlation analyses of intracellular and FP data depicted in Figure 10. Two minute periods (including the ones presented in Fig. 10) were analyzed with the sequential correlation method. See description in text. 
three recording sites. Intracortical infusion of lidocaine in the middle suprasylvian gyrus inactivated the EEG close to the cannula and disrupted the synchrony between single neurons as well as FPs recorded from the anterior and posterior suprasylvian sites (see epochs underlined by dotted lines in which depolarizations and hyperpolarizations in the posterior suprasylvian neuron corresponded to opposite events in the anterior neuron). However, the local synchronization processes between the impaled neurons and the close FPs were preserved both anteriorly and posteriorly.

The sequential correlation analyses of data depicted in Figure 10 are shown in Figure 11. The synchrony between intracellular potentials and the neighboring FPs is demonstrated by deep, well aligned, central negative peaks. The black surface in the left contour map, close to zero time-lag (only the anterior impalement and the adjacent FP are shown here), is expected because intracellular potentials and depth-cortical FPs are opposite in phase. They were not affected by the infusion of lidocaine. By contrast, the cross-correlations between the two intracellular activities (middle column) as well as between the respective FPs (right column) evolved from a coherent pattern in the control period to a disorganized sequence of peaks and valleys, characteristic for desynchronized activities. According to the SyCo, the synchrony between intracellular potentials dropped from 0.59 to 0.15 , and between FPs from 0.74 to 0.25 . This stood in contrast with the fact that SyCo on each side of the lidocaine injection remained around 0.8 .

\section{Effects of intracortical transections upon the synchrony of slow oscillation}

Coronal cuts were performed at various antcrior plancs, from 4 to 7 , in the left hemisphere. The transections involved the suprasylvian gyrus or, in other animals, they extended from the marginal to the ectosylvian gyrus (Fig. 12 and cortex figurine in Fig. 13). Similar results were obtained in both types of experiments. Most cuts were about $2 \mathrm{~mm}$, up to and including deep cortical layers. Even when the cuts reached $5 \mathrm{~mm}$ (Fig. 12B), protruding deeply into the white matter, they never reached subcortical structures.

Figure 13 depicts the effects of large, marginal-to-ectosylvian, transections upon the intracortical synchronization of the slow oscillation. Two couples of electrodes recorded the FPs in the suprasylvian gyrus, rostrally (1-2) and caudally (3-4) from the transection plane. The sequential cross-correlations between adjacent electrodes during a control period, before performing the transection, showed synchronous activities with high coefficient values (SyCo 0.91 in CROSS 1-2, and 0.9 in CROSS 3-4). These features remained unaffected by the transection, up to $6 \mathrm{hr}$ later. On the other hand, the synchrony between the anterior and posterior sites (CROSS 2-4) changed from the control period (SyCo $=0.73$ ) to the posttransection period (SyCo $=0.36$ ). Similar values were obtained for CROSS 1-3 (data not shown). Each sequential cross-correlation in Figure 13 is accompanied (at its right) by the $\tau$-diagram of the sequential time-lags seen from the same view angle as the correlations (see again the method at bottom right in Fig. 1). The dotted line in the $\tau$-diagram marks zero time-lag. It results that the dispersion of correlation peaks was increased after transecting the cortex between rostral and caudal recording sites (CROSS 2-4), while it did not significantly change in recordings on the same side of the cut (CROSS 1-2 and CROSS 3-4). The effects of the transection were therefore similar to the effects of cortical inactivation by lidocaine.
Six hours after the transection was performed, the synchrony between the same distant foci (2-4) recovered partially (see bottom right). Time-lags tended to align again and their dispersion diminished. The SyCo reached to 0.5 (lower than in the control period, but higher than 2 min after the transection).

We calculated the SyCo from all 34 recording sessions, before and after lidocaine inactivation or transection. During control periods, SyCo between closely located recording sites (about 1 $\mathrm{mm})$ was $0.91 \pm 0.05$, while it was lower $(0.67 \pm 0.09)$ between distantly located foci (about $15 \mathrm{~mm}$ ). Intracortical infusion of lidocaine or transections did not affect significantly the SyCo between leads placed on the same side of the reversible inactivation or transection $(0.9 \pm 0.06)$, but significantly decreased the SyCo between channels separated by the inactivated or transected scctor $(0.26 \pm 0.08)$. This represents a drop in synchrony of $61 \%$.

\section{Intergyral paths may provide compensation for synchrony among disconnected foci}

The above data show that, in spite of the diminished synchrony after reversible inactivation or transection, synchrony was not completely abolished and recovery of control patterns was observed at long time intervals after disconnection. To elucidate possible sources for the compensatory mechanisms underlying these phenomena, we placed arrays of bipolar electrodes crossing over adjacent gyri. Figure 14 depicts data from an experiment in which recordings of multisite FPs were performed in the suprasylvian and marginal gyri (see cortex figurine). Lidocaine was injected in the suprasylvian gyrus, at the site indicated by arrow in the bottom scheme, and the inactivation extended up to electrode 2, $1 \mathrm{~mm}$ apart. Only the synchrony between anterior and posterior leads in the suprasylvian gyrus ( 1 and 3 , respectively) was affected, and it was accompanied by a SyCo drop from 0.74 to 0.21 , whereas the other cross-correlations, including that between the rostral suprasylvian lead 1 and the posterior lead 5 from the marginal gyrus, were not much affected (see Discussion).

\section{Discussion}

We have shown that (1) the rostral and caudal parts of the association suprasylvian gyrus are reciprocally linked through short-latency excitatory projections; (2) reversible inactivation or transection at the middle suprasylvian level disrupts the coherence of the slow oscillation between distant, anterior and posterior, sites of the gyrus, while leaving intact local (anterior as well as posterior) synchronizing processes; and (3) the loss of long-range coherence is not permanent, as synchrony recovers a few hours after disconnection. These data demonstrate that fibers traveling within the suprasylvian gyrus and/or the underneath white matter are necessary and sufficient to sustain the synchronization of the slow rhythm between distant cortical sites. We suggest that intergyral paths and/or corticothalamic loops exert vicarious functions after disconnection of synaptic linkages within the suprasylvian gyrus.

\section{Some methodological issues}

It was previously reported that the frequency of the slow cortical oscillation is $0.2-0.5 \mathrm{~Hz}$ under urethane anesthesia and that it increases to $0.5-0.9 \mathrm{~Hz}$ by ketamine administration (Steriade et al., 1993b), due to the blockage of NMDA receptors, thus significantly reducing the duration of the depolarizing phase of the oscillation. The frequency range of $0.5-0.9 \mathrm{~Hz}$ was also ob- 

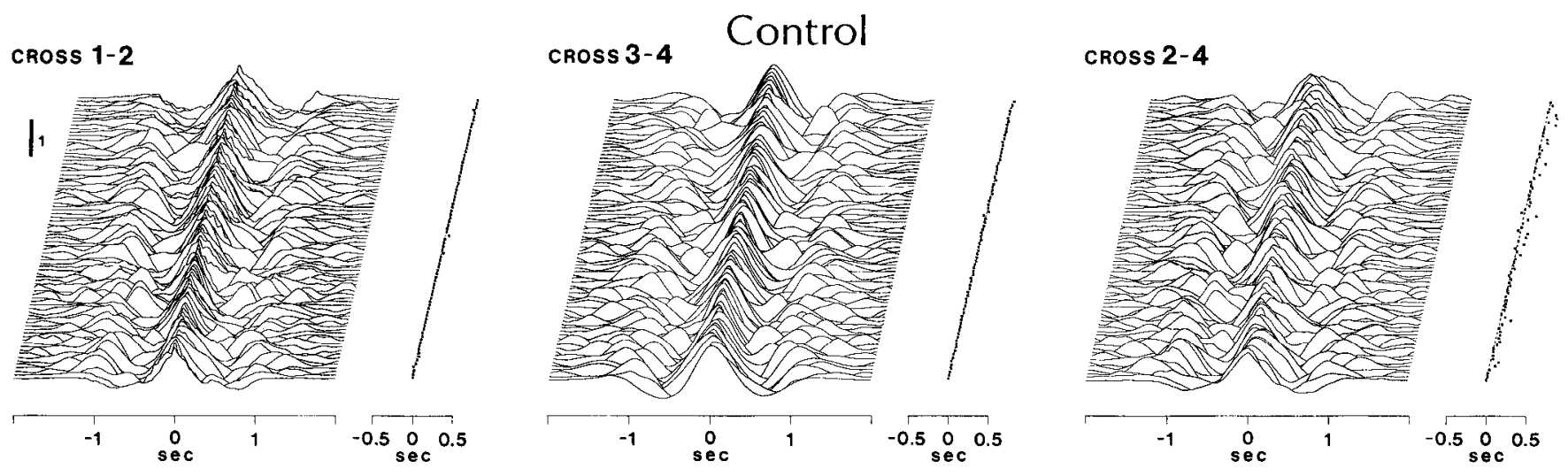

\section{2 min after transection}
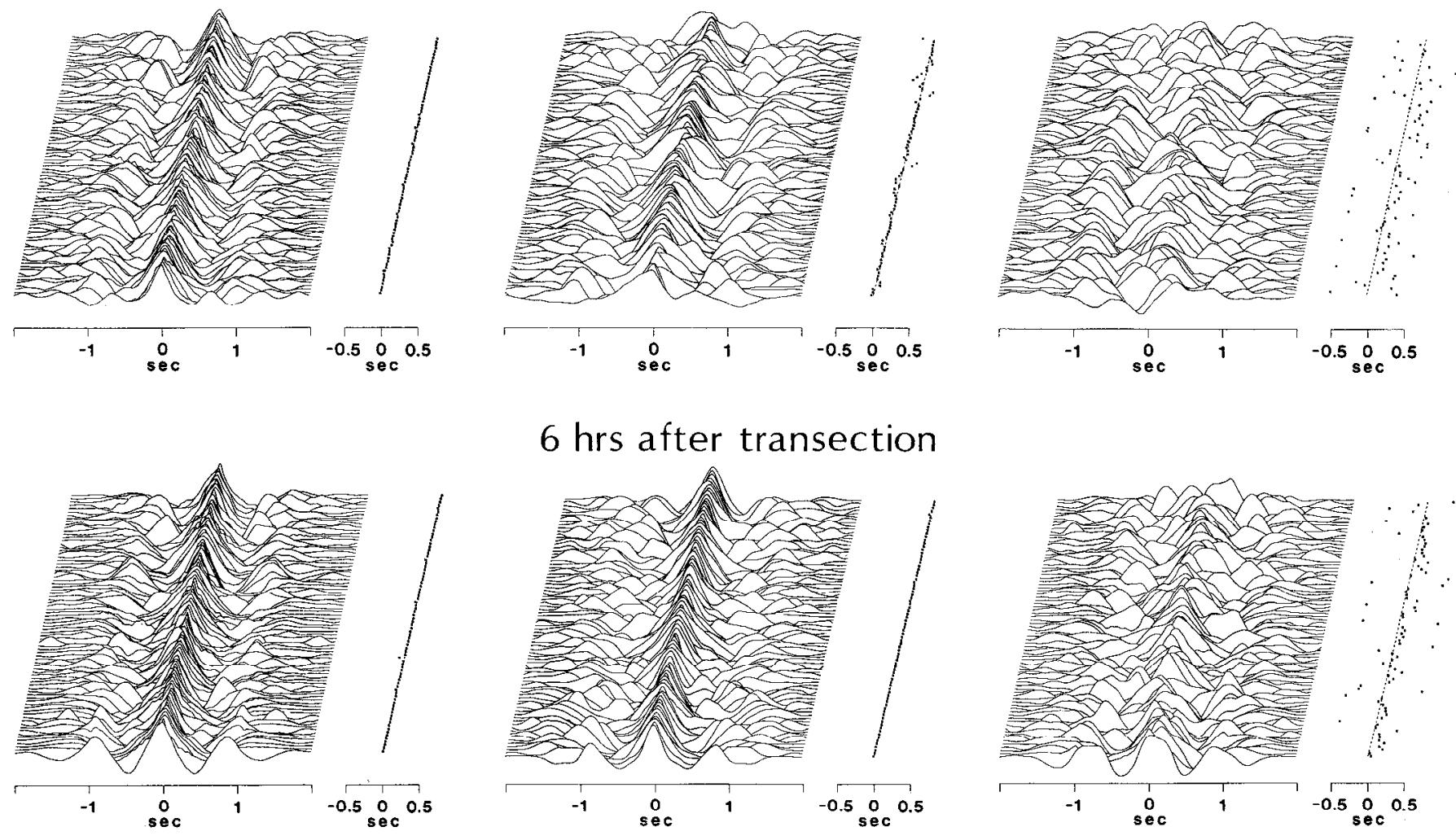

\section{6 hrs after transection}
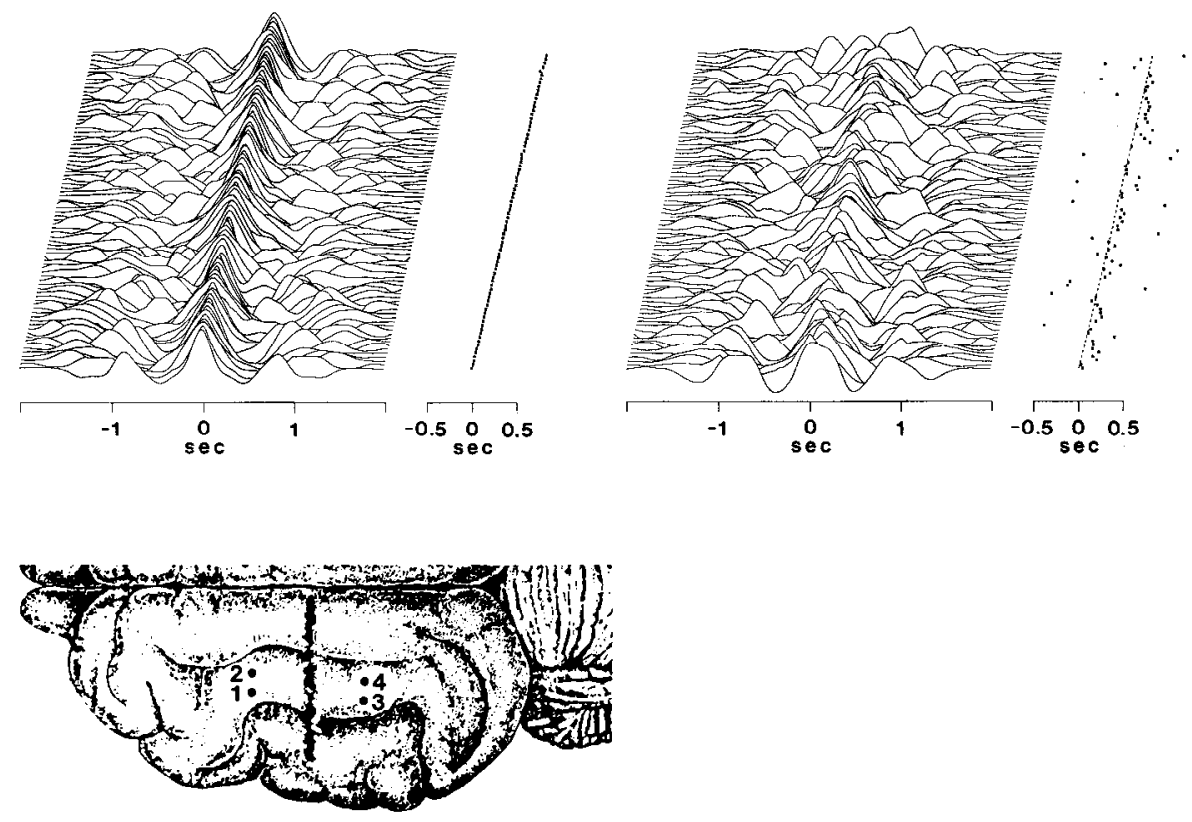

Figure 13. Effect of a coronal transection, from marginal to ectosylvian gyri, upon intracortical synchrony. Two couples of electrodes recorded the FPs in the suprasylvian gyrus, rostrally $(1-2)$ and caudally $(3-4)$ from the transection plane. See text for description of results from sequential cross-correlations and $\tau$ diagrams.

served in the present study conducted under ketamine and $x y-$ lazine anesthesia. The general pattern of the slow rhythm remained the same, with periodic sequences of prolonged depolarizations and long-lasting hyperpolarizations. The former are associated with negativities of depth-cortical FPs, the latter are related to íocal positivities (Contreras and Steriade, 1995; see also Fig. 10 in the present article). The use of ketamine and xylazine anesthesia may increase the degree of synchronization, as compared to that occurring during natural sleep, probably because xylazine, an $\alpha 2$ receptor agonist, increases a $\mathrm{K}^{+}$con- 


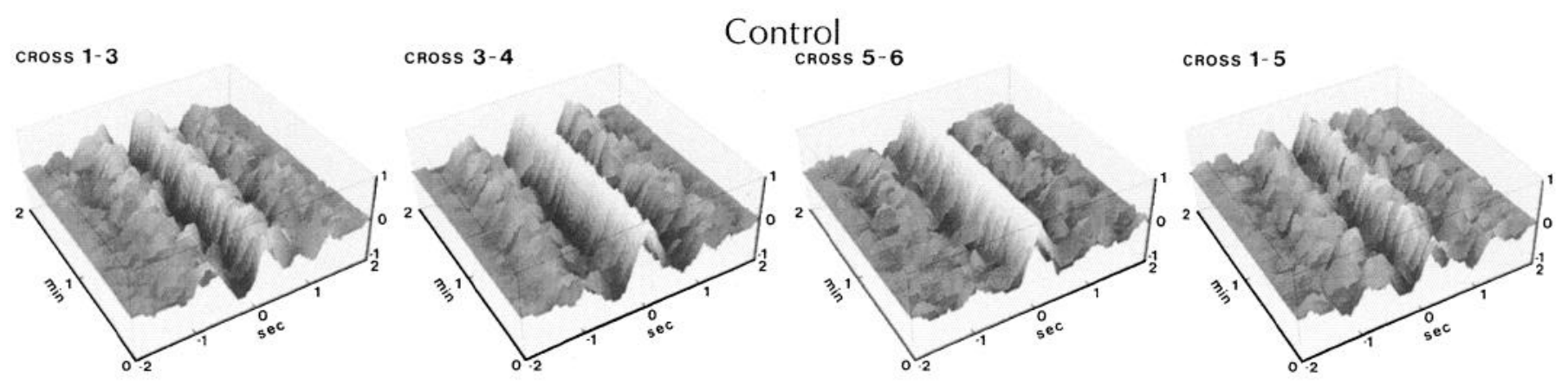

After lidocaine
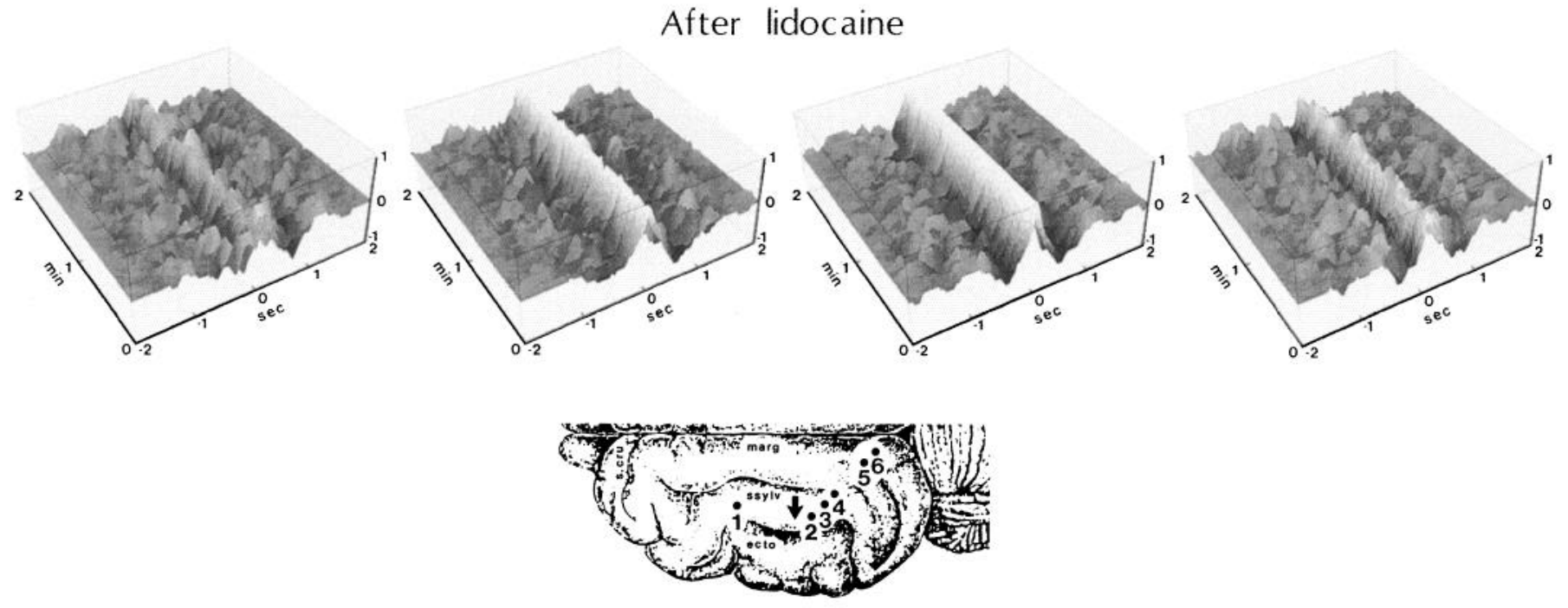

Figure 14. Possible intergyral projections functioning for compensation of synchrony between functionally disconnected foci. Recordings of FPs from the six indicated foci in the suprasylvian and posterior marginal gyri. Lidocaine injection $(1 \mu \mathrm{l}, 4 \%)$ marked by arrow. See text for description.

ductance in a variety of central structures (Nicoll et al., 1990) and the rhythmic long-lasting hyperpolarizations mediated by $\mathrm{K}^{+}$currents are priming events toward the corticothalamic synchronization underlying the slow oscillation (Steriade et al., 1993a, 1994b; Contreras and Steriade, 1995). Ketamine is placed among the most effective pharmacological tools in inducing slow-wave sleep patterns over the background of a wake state (Feinberg and Campbell, 1993).

We emphasize that slow oscillatory patterns, similar to those obtained under different anesthetic conditions, have been observed intracellularly in undrugged animals with isolated forebrain by mesencephalic transections (Steriade et al., 1993c) and, at the EEG level, in naturally sleeping cats and humans (Steriade et al., 1993b). Recent extracellular studies in chronically implanted, behaving animals support the presence of the slow rhythm during natural EEG-synchronized sleep, with similar patterns as those observed under anesthesia (unpublished data).

The intracortical spread of lidocaine and the time-course of its action have been assessed autoradiographically (Martin, 1991). In that paper, the maximal radius of lidocaine spread was about $1.7 \mathrm{~mm}$, the action was achieved within the first $20 \mathrm{~min}$ postinjection, and the width decreased after 30-60 min. As we aimed at obtaining clear-cut effects appearing immediately or a few minutes after lidocaine infusion (see Figs. 7, 8), in order to avoid changes in the global state of brain electrical activity, higher concentrations and volumes were used in our experiments. This is probably the reason why the recovery after li- docaine inactivation occurred after a few hours, compared with 15-45 min in some behavioral studies of motor systems (Demer and Robinson, 1982; Martin and Ghez, 1988).

\section{Substrates of intracortical synchronization of the slow oscillation}

Although the slow oscillation is not evenly distributed within the neocortex and the signs of underlying synchrony are most obvious within and between suprasylvian association areas 5 and 7 or visual association areas $18 / 19$ and 21 , a long-range synchronization between cortical fields as distant as the motor and visual cortices was also described (Amzica and Steriade, 1995). Most adjacent and many distant neocortical territories are linked by means of reciprocal projections. The horizontal projections of pyramidal cells' axons, spanning from 2 up to $8 \mathrm{~mm}$, have been emphasized in the visual cortex where they allow communication between neurons having widely separated receptive fields (Gilbert and Wiesel, 1983; Mason et al., 1991; Gilbert, 1992; Albowitz and Kuhnt, 1993). A basically similar type of connectivity also seems to characterize the somatosensory (Jones et al., 1978; Cauller and Connors, 1994), auditory (Imig and Reale, 1981), and motor (Keller, 1993) cortical areas, as well as the projections between sensory and motor fields (Avendaño et al., 1992).

The cat suprasylvian gyrus, within which most of the present experiments were conducted, has reciprocal projections with adjacent cortical areas and massive intragyral connections. It is 
now established that association areas integrate polymodal signals from heterogeneous sources. In the case of the parietal association areas, the main extrinsic cortical projections arise in the somatosensory areas (Jones and Powell, 1969; Jones et al., 1978; Burton and Kopf, 1984), primary and association visual fields (Pandya et al., 1981; Squatrito et al., 1981a,b), as well as motor, insular, and prefrontal areas (Jones et al., 1978; Mesulam and Mufson, 1982; Godschalk et al., 1984). The cortical suprasylvian areas 5 and 7 also receive afferents from the thalamus, mainly arising in the rostral intralaminar and lateroposterior-pulvinar nuclei (Graybiel, 1972; Hendry et al., 1979; Avendaño et al., 1985). Their neurons send monosynaptic, high-security projections to cortex and receive in turn inputs from the same areas (Steriade et al., 1977, 1993c). Areas 5 and 7 are interconnected through abundant intragyral suprasylvian fiber bundles (Grüner et al., 1974; Avendaño et al., 1988) and project to motor cortical areas, thus probably transmitting convergent sensory information used for voluntary movements (Babb et al., 1984).

Horizontal axons provide strong input to dendrites of pyramidal cells in layers $\mathrm{II}-\mathrm{IV}$ within a distant column (Aroniadou and Keller, 1993; Coogan and Burkhalter, 1993). As to the horizontal layer I input, it may trigger active currents along the apical dendrites of pyramidal neurons that would amplify the EPSPs in their way to the soma (Cauller and Connors, 1994). While most horizontal intracortical connections are provided by excitatory axons arising in pyramidal-shaped neurons, a similar excitatory outcome may result from lateral disinhibitory actions arising from contacts between GABAergic basket cells and other inhibitory local-circuil neurons (Kisvárday et al., 1993). Earlier data on the basic patterns of intra- and intercolumnar interactions resulting from common excitation and serial inhibition in sensory and motor cortical areas have been reviewed by Fetz et al. (1991).

The intracortical excitatory projections are mediated by both NMDA and non-NMDA receptors (Thomson, 1986; Sutor and Hablitz, 1989a,b; Hwa and Avoli, 1992) that are found on both long-axoned and local-circuit neurons (Kawaguchi, 1993). The NMDA components, which are blocked at a hyperpolarized membrane potential (Sutor and Hablitz, 1989b; Takahashi and Ogawa, 1991), are likely to be involved in the augmenting cortical responses to low-frequency repetitive stimulation as our previous study (Nuñez et al., 1993) showed that the longer-latency EPSPs, which underlie the incremental component of augmenting-type responses, are decreased upon hyperpolarization. Although the involvement of thalamic neurons in incremental responses cannot be ruled out in a brain-intact preparation, the augmenting responses evoked in the present study by stimulating distant sites within the suprasylvian gyrus (see Figs. 3, 6) are probably generated intracortically since similar incremental responses were elicited after extensive destruction of the thalamus (see Fig. 7 in Nuñez et al., 1993). Previous work in somatosensory and visual systems also indicated that the intracortical circuitry can underlie incremental responses, as shown by augmentation to repetitive stimuli applied to the white matter in thalamic-lesioned animals (see Fig. 8 in Morin and Steriade, 1981; also Ferster and Lindström, 1983). This is further supported by changes in augmenting responses after the intracortical inactivation procedure used in the present experiments (see Fig. 6).

All these intragyral and extrinsic cortical connections explain the strong oscillatory propensity of areas 5 and 7 (present experiments), the presence of coherent slow oscillations in area 5 , primary and association visual areas (see Fig. 14), and the survival of the intracellularly recorded slow oscillation after total lesions of intralaminar and lateroposterior-pulvinar thalamic nuclei (Steriade et al., 1993c). The massive projection from area 7 to area 5 (Kawamura, 1973; Avendaño et al., 1988) is the anatomical substrate of the short-latency, posterior-to-anterior suprasylvian EPSPs revealed in the present experiments (see Figs. 2-6). In a previous study (Amzica and Steriade, 1995) we similarly pointed to a preferential traveling of oscillatory waves in a posterior-to-anterior direction, with the firing of more caudal neurons preceding that of rostral ones in $70 \%$ of cases.

\section{Synchronization mechanisms of the slow oscillation and the recovery of coherence between distant sites after disconnection of intracortical synaptic linkages}

Our results support the hypothesis that the slow oscillation is an emergent property of intracortical networks. We based this assumption on the presence of the slow rhythm in intracellularly recorded areas 5 and 7 neurons after total electrolytic or excitotoxic lesions of related thalamic nuclei (Steriade et al., 1993c). In the same study, however, thalamic stimulation was effective in altering the cortical slow rhythm and in transforming its frequency into faster oscillations. The cortical slow oscillation is reflected in thalamic reticular and thalamocortical cells (Steriade et al., 1993d). The fact that prolonged and cyclic hyperpolarizations, within the frequency range of the slow rhythm, are observed in cortical, thalamic reticular, and thalamocortical neurons, and that synchronous hyperpolarizations in simultaneously recorded cortical and thalamic neurons are coincident with the appearance of overt signs of EEG synchronization led to the hypothesis that this generalized inhibitory process is a priming factor in the synchronization of slow oscillations during EEGsynchronized sleep (Steriade et al., 1994b; Contreras and Steriade, 1995). In fact, the suppression of synchronization in brain electrical activity may be achieved by a blockage of long-lasting hyperpolarizations (Steriade et al,, 1993a, 1994b). In the case of an oscillation with such long periods ( $>1 \mathrm{sec}$ ), the term synchronization implicates that long time-lags, below one order of magnitude of the oscillation period, may be seen between oscillating cortical cells recorded from distant areas (Amzica and Steriade, 1995). Such time-lags in cross-correlograms are ascribed either to inhibitory-rebound processes within the cortex and/or the fact that dorsal thalamic cells may discharge lowthreshold spike-bursts, transferred back to cortex, with long delays (100 msec or more) after their prolonged IPSPs (see Fig. $8 C$ in Contreras and Steriade, 1995; also Fig. 6 in Amzica and Steriade, 1995).

Could it be envisaged that the synchronization of the slow oscillation is entirely supported by the intracortical circuitry? We attempted to inactivate various thalamic regions by means of lidocaine and were still able to record synchronous activities from multiple sites in related neocortical areas (unpublished data). However, this inactivation procedure affected rather limited $(1-2 \mathrm{~mm})$ thalamic territories. The presence of thalamic inputs arising from different nuclei and converging upon the same cortical area(s) makes difficult a definite conclusion. Although the slow cortical oscillation survived extensive thalamic lesions (Steriade et al., 1993c), its synchronization may involve thalamic inputs. In particular, rostral intralaminar thalamic nuclei that reflect the slow oscillation, project widely over the neocortex, and efficiently transfer thalamic rhythms at the cortical level 
(Steriade et al., 1990) may be involved in the coherency of slow oscillation within corticothalamocortical loops.

The present status of our hypotheses concerning the synchronization of the slow oscillation and the compensation process after the loss of coherence by disrupting intracortical synaptic linkages is that (1) disconnected cortical neuronal pools can independently generate the slow oscillation at different frequencies, but (2) intact connections between neuronal assemblies regularize the oscillation within the same frequency range, is shown in the FP (Fig. 7) and intracellular (Fig. 10) recordings, as well as in FFT analyses (Fig. 9). As illustrated in these figures, both anterior and posterior suprasylvian neurons continued to oscillate after their disconnection. However, not only the coherence of their slow oscillation was disrupted by inactivating the intrasuprasylvian connections within the middle part of the gyrus, but the oscillatory patterns recorded from each (anterior or posterior) side were altered as compared to the control period (see intracellular and FP recordings from the anterior site in Fig. 10) and their rhythms dramatically changed (see the decreased frequency within the posterior site in Fig. 9).

We have previously assumed that the prolonged hyperpolarizations that prime the synchronization of the slow oscillation are due to $\mathrm{Ca}^{2+}$-dependent $\mathrm{K}^{+}$currents and/or disfacilitation processes (Steriade et al., 1993a, 1994b; Contreras and Steriade, 1995). The involvement of $\mathrm{Ca}^{2+}$-dependent $\mathrm{K}^{+}$currents was postulated in view of the fact that setting into action ascending activating cholinergic systems erases these hyperpolarizations (Steriade et al., 1993a) and ACh suppresses $g_{\mathrm{K}(\mathrm{Ca})}$ (McCormick and Williamson, 1989; Schwindt et al., 1989). The alternative, nonexclusive mechanism of an avalanche disfacilitation in corticothalamic circuits during the prolonged hyperpolarizations may operate not only during the normal slow sleep oscillation, but also during an extreme degree of disconnection termed EEG burst-suppression, as a few volleys may succeed in restoring the patterns of brain electrical activity (see Fig. 7 in Steriade et al., 1994a). Thus, subtle changes in the network lead to start again the oscillatory cycles and to synchronize local as well as distant neuronal pools. During the slow oscillation, such changes may be represented by EPSPs occurring toward the end of the prolonged hyperpolarizations, leading to rebound activities that could eventually synchronize the whole network. That, indeed, the depolarizing phase of the oscillation is initiated by postinhibitory rebound excitations in cortical neurons was repeatedly depicted in our intracellular studies (see, e.g., Fig. $7 A$ in Steriade et al., 1993b; also Fig. $2 A$ in Steriade et al., 1994a).

The thalamus may be implicated in the vicariant functions that would account for the recovery of synchronization following cortical disconnection. This should be further investigated by using functional or morphological removal of larger thalamic territories than those we succeeded to inactivate until now (see above). However, accumulating experimental evidence seems to indicate that intrahemispheric and/or callosal projections are more efficient than thalamocortical ones to account for cortical remodelling during functional recovery (Miller et al., 1991; Darian-Smith and Gilbert, 1994).

The intracortical synchronization processes demonstrated in the present study are certainly implicated during resting sleep, as our experiments in progress show their presence during natural states of vigilance in behaving animals, but similar processes probably underlie the dynamic coupling between neocortical cells during transition from normally synchronized sleep states to paroxysmal activities of epileptic type. Multisite, extra- and intracellular recordings from cortical suprasylvian and marginal areas 5, 7, 18, and 21 demonstrated that the buildup of seizures obeys the rule of synaptic circuits, sequentially distributed through short- and long-range synaptic linkages, with a progressive increase in the degree of neuronal synchrony from the preseizure sleep patterns to the early and late stages of seizures (Steriade and Amzica, 1994). The participation of thalamic neurons in the devclopment of paroxysmal activities (Steriade and Contreras, 1995) further enhances the synchronization processes and contributes to their spread and generalization.

\section{References}

Albowitz B, Kuhnt U (1993) The contribution of intracortical connections to horizontal spread of activity in the neocortex as revealed by voltage sensitive dyes and a fast optical recording method. Eur J Neurosci 5:1349-1359.

Amzica F, Steriade M (1995) Short- and long-range neuronal synchronization of the slow $(<1 \mathrm{~Hz})$ cortical oscillation. J Neurophysiol 73 : 20-39.

Aroniadou VA, Keller A (1993) The patterns and synaptic properties of horizontal intracortical connections in the rat motor cortex. J Neurophysiol 70:1553-1569.

Avendaño C, Rausell E, Reinoso-Suárez F (1985) Thalamic projections to areas $5 \mathrm{a}$ and $5 \mathrm{~b}$ of the parietal cortex in the cat: a retrograde horseradish peroxidase study. J Neurosci 5:1446-1470.

Avendaño C, Rausell E, Perez-Aguilar D, Isorna S (1988) Organization of the association cortical afferent connections of area 5: a retrograde tracer study in the cat. J Comp Neurol 278:1-33.

Avendaño C, Isla AJ, Rausell E (1992) Area 3a in the cat. II. Projections to the motor cortex and their relations to other corticocortical connections. J Comp Neurol 321:373-386.

Babb RS, Waters RS, Asanuma H (1984) Corticocortical connections to the motor cortex from the posterior parietal lobe (areas $5 \mathrm{a}, 5 \mathrm{~b}, 7$ ) in the cat demonstrated by the retrograde axonal transport of horseradish peroxidase. Exp Brain Res 54:476-484.

Bendat JS, Piersol AG (1980) Engineering applications of correlation and spectral analysis. New York: Wiley.

Burton H, Kopf EM (1984) Ipsilateral cortical connections from the second and fourth somatic sensory areas in the cat. J Comp Neurol 225:527-553.

Cauller LJ, Connors BW (1994) Synaptic physiology of horizontal afferents in layer I in slices of rat SI neocortex. J Neurosci 14:751762.

Clare MH, Bishop GH (1954) Responses from an association area secondarily activated from optic cortex. J Neurophysiol 17:271-277.

Contreras D, Steriade M (1995) Cellular basis of EEG rhythms: a study of dynamic corticothalamic relationships. J Neurosci 15:604-622.

Coogan TA, Burkhaiter A (1993) Hierarchical organization of areas in rat visual cortex. J Neurosei 13:3749-3772.

Darian-Smith C, Gilbert CD (1994) Axonal sprouting accompanies functional reorganization in adult cat striate cortex. Nature 368:737740.

Demer JL, Robinson DA (1982) Effects of reversible lesions and stimulation of olivocerebellar system on vestibuloocular reflex plasticity. J Neurophysiol 47:1084-1107.

Feinberg I, Campbell IG (1993) Ketamine administration during waking increases delta EEG intensity in rat sleep. Neuropsychopharmacology $9: 41-48$.

Ferster D, Lindström S (1983) An intracellular analysis of geniculocortical connectivity in area 17 of the cat. J Physiol (I ond) 342:181215

Fetz E, Toyama K, Smith W (1991) Synaptic interactions between cortical neurons. In: Cerebral cortex, Vol 9 (Peters $\Lambda$, Jones EG, eds), pp 1-47. New York: Plenum.

Gilbert CD (1992) Horizontal integration and cortical dynamics. Neuron $9: 1-13$.

Gilbert CD, Wiesel TN (1983) Clustered intrinsic connections in cat visual cortex. J Neurosci 3:1116-1133.

Godschalk M, Lemon RN, Kuypers HGJM, Ronday HK (1984) Cortical afferents and efferents of monkey postarcuate area: an anatomical and electrophysiological study. Exp Brain Res 56:410-424.

Graybiel AM (1972) Some fiber pathways related to the posterior thalamic region in the cat. Brain Behav Evol 6:363-393. 
Grüner JE, Hirsch JC, Sotelo C (1974) Ultrastructural features of the isolated suprasylvian gyrus in the cat. J Comp Neurol 154:1-28.

Hassler R, Muhs-Clement K (1964) Architektonischer Aufbau des sensomotorischen und parietalen Cortex der Katze. J Hirnforsch 6:377422.

Heath CJ, Jones EG (1971) The anatomical organization of the suprasylvian gyrus of the cat. Ergeb Anat Entwicklungsgesch 45:4-64.

Hendry SHC, Jones EG, Graham J (1979) Thalamic relay nuclei for cerebellar and certain related fiber systems in the cat. J Comp Neurol 185:679-714.

Hwa GGC, Avoli M (1992) Excitatory postsynaptic potentials recorded from regular-spiking cells in layers II/III of rat sensorimotor cortex. J Neurophysiol 67:728-737.

Imig TJ, Reale RA (1981) Ipsilateral corticocortical projections related to binaural columns in cat primary auditory cortex. J Comp Neurol 203:1-14.

Jones EG, Powell TPS (1969) Connexions of the somatic sensory cortex of the rhesus monkey. I. Ipsilateral cortical connexions. Brain 92 ; 477-502.

Jones EG, Coulter JD, Hendry SHC (1978) Intracortical connectivity of architectonic fields in somatic sensory, motor and parietal cortex of monkeys. J Comp Neurol 181:291-348.

Kawaguchi Y (1993) Groupings of nonpyramidal and pyramidal cells with specific physiological and morphological characteristics in rat frontal cortex. J Neurophysiol 69:416-431.

Kawamura K (1973) Corticocortical fiber connections of the cat cerebrum. II. The parietal region. Brain Res 51:23-40.

Keller A (1993) Intrinsic synaptic organization of the motor cortex. Cereb Cortex 3:43-41.

Kisvárday ZF, Beaulieu C, Eysel UT (1993) Network of GABAergic large basket cells in cat visual cortex (area 18): implication for lateral disinhibition. J Comp Neurol 32\%:398-415.

Martin JH (1991) Autoradiographic estimation of the extent of reversible inactivation produced by microinjection of lidocaine and muscimol in the rat. Neurosci Lett 127:160-164.

Martin JH, Ghez C (1988) Red nucleus and motor cortex: parallel motor systems for the initiation and control of skilled movement. Behav Brain Res 28:217-223.

Mason A, Nicoll A, Stratford K (1991) Synaptic transmission between individual pyramidal neurons of the rat visual cortex in vitro. J Neurosci 11:72-84.

McCormick DA, Williamson A (1989) Convergence and divergence of neurotransmitter action in human cerebral cortex. Proc Natl Acad Sci USA 86:8098-8102.

Mesulam MM, Mufson EJ (1982) Insula of the Old World monkey. III. Efferent cortical output and comments on function. J Comp Neurol 212:38-52.

Miller B, Windrem MS, Finlay BL (1991) Thalamic ablations and neocortical development: alterations in thalamic and callosal connectivity. Cereb Cortex 1:241-261.

Montero VM (1981) Topography of the corticu-cortical connections from the striate cortex in the cat. Brain Behav Evol 18:194-218.

Morin D, Steriade M (1981) Development from primary to augmenting responses in the somatosensory system. Brain Res 205:49-66.

Nicoll RA, Malenka RC, Kauer JA (1990) Functional comparison of neurotransmitter receptor subtypes in mammalian central nervous system. Physiol Rev 70:513-565.

Nuñez A, Amzica F, Steriade M (1993) Electrophysiology of cat association cortical cells in vivo: intrinsic properties and synaptic responses. J Neurophysiol 70:418-430.

Pandya DN, Van Hoessen GW, Mesulam MM (1981) Efferent connec- tions of the cingulate gyrus in the rhesus monkey. Exp Brain Res 42: 319-330.

Schwindt PC, Spain WJ, Foehring RC, Chubb MC, Crill WE (1989) Slow conductances in neurons from cat sensorimotor cortex in vitro and their role in slow excitability changes. J Neurophysiol 59:450467.

Sherk H (1986) Location and connections of visual cortical areas in the cat's suprasylvian sulcus. J Comp Neurol 247:1-31.

Squatrito S, Galletti C, Battaglini PP, Riva Sanseverino E (1981a) Bilateral cortical projections from cat visual areas 17 and 18. Arch Ital Biol 119:1-20.

Squatrito S, Galletti C, Battaglini PP, Riva Sanseverino E (1981b) An autoradiographic study of bilateral cortical projections from cat area 19 and lateral suprasylvian visual area. Arch Ital Biol 1 19:21-42.

Steriade M (1978) Cortical long-axoned cells and putative interneurons during the sleep-waking cycle. Behav Brain Sci 1:465-514.

Steriade M (1993) Cellular substrates of brain rhythms. In: Electroencephalography: basic principles, clinical applications, and related fields (Niedermeyer E, Lopes da Silva F, eds), pp 27-62. Baltimore: Williams and Wilkins.

Steriade M, Amzica F (1994) Dynamic coupling among neocortical neurons during evoked and spontaneous spike-wave seizure activity. J Neurophysiol 72:2051-2069.

Steriade M, Contreras D (1995) Relations between cortical and thalamic cellular events during transition from sleep patterns to paroxysmal activity. J Neurosci 15:623-643.

Steriade M, Diallo A, Oakson G, White-Guay B (1977) Some synaptic inputs and ascending projections of lateralis posterior thalamic neurons. Brain Res 131:39-53.

Steriade M, Jones EG, Llinás RR (1990) Thalamic oscillations and signaling. New York: Wiley.

Steriade M, Amzica F, Nuñez A (1993a) Cholinergic and noradrenergic modulation of the slow $(\approx 0.3 \mathrm{~Hz})$ oscillation in neocortical cells. $J$ Neurophysiol 70:1385-1400.

Steriade M, Nuñez A, Amzica F (1993b) A novel slow $(<1 \mathrm{~Hz})$ oscillation of neocortical neurons in vivo: depolarizing and hyperpolarizing components. J Neurophysiol 70:3252-3265.

Steriade M, Nuñez A, Amzica F (1993c) Intracellular analysis of relations between the slow $(<1 \mathrm{~Hz})$ neocortical oscillation and other sleep rhythms of the electroencephalogram. J Neurosci 13:32663283.

Steriade M, Contreras, Curró Dossi R, Nuñez A (1993d) The slow $(<1$ $\mathrm{Hz}$ ) oscillation in reticular thalamic and thalamocortical neurons: scenario of sleep rhythm generation in interacting thalamic and neocortical networks. J Neurosci 13:3284-3299.

Steriade M, Amzica F, Contreras D (1994a) Cortical and thalamic cellular correlates of electroencephalographic burst-suppression. Electroencephalogr Clin Neurophysiol 90:1-16.

Steriade M, Contreras D, Amzica F (1994b) Synchronized sleep oscillations and their paroxysmal developments. Trends Neurosci 17:199_ 208.

Sutor B, Hablitz JJ (1989a) EPSPs in rat neocortical neurons in vitro. I. Electrophysiological evidence for two distinct EPSPs. J Neurophysiol 61:607-620.

Sutor B, Hablitz JJ (1989b) EPSPs in rat neocortical neurons in vitro. II. Involvement of $N$-methyl-D-aspartate receptors in the generation of EPSPs. J Neurophysiol 61:621-634.

Takahashi Y, Ogawa T (1991) Composition of the excitatory postsynaptic potentials recorded from rat visual cortex neurons in layer II/ III: an in vitro electrophysiological and pharmacological study. Tokohu J Exp Med 164:213-221.

Thomson AM (1986) A magnesium-sensitive postsynaptic potential in rat cerebral cortex resembles neuronal responses to $\mathrm{N}$-methylaspartate. J Physiol (Lond) 370:531-549. 